\title{
STABLE NLS SOLITONS IN A CUBIC-QUINTIC MEDIUM WITH A DELTA-FUNCTION POTENTIAL
}

\author{
FRANÇOIS GENOUD, BORIS A. MALOMED, AND RADA M. WEISHÄUPL
}

\begin{abstract}
We study the one-dimensional nonlinear Schrödinger equation with the cubic-quintic combination of attractive and repulsive nonlinearities, and a trapping potential represented by a delta-function. We determine all bound states with a positive soliton profile through explicit formulas and, using bifurcation theory, we describe their behavior with respect to the propagation constant. This information is used to prove their stability by means of the rigorous theory of orbital stability of Hamiltonian systems. The presence of the trapping potential gives rise to a regime where two stable bound states coexist, with different powers and same propagation constant.
\end{abstract}

\section{INTRODUCTION}

In this paper we study the one-dimensional nonlinear Schrödinger (NLS) equation with the cubic-quintic (CQ) combination of attractive and repulsive nonlinearities, and a trapping potential represented by a delta-function:

$$
i \psi_{z}=-\psi_{x x}-\epsilon \delta(x) \psi-2|\psi|^{2} \psi+|\psi|^{4} \psi, \quad(x, z) \in \mathbb{R}^{2},
$$

for complex $\psi=\psi(x, z)$, and $\epsilon>0$. The objective of the analysis is the existence and stability of localized bound states, in the form of $\psi(x, z)=\mathrm{e}^{i k z} u(x)$, with $u(x)>0$ satisfying the respective stationary equation:

$$
u^{\prime \prime}-k u+\epsilon \delta(x) u+2 u^{3}-u^{5}=0, \quad x \in \mathbb{R} .
$$

Here and henceforth, ' stands for differentiation with respect to $x$. Denoting by $\langle\cdot, \cdot\rangle$ the duality product between $H^{-1}(\mathbb{R})$ and $H^{1}(\mathbb{R})$ - and recalling that $H^{1}(\mathbb{R}) \subset C(\mathbb{R}) \cap L^{\infty}(\mathbb{R})$ - , the potential $\delta$ appearing in the soliton equation (1.2) is the Dirac distribution at $x=0$, defined by $\langle\delta, u\rangle=u(0)$ for all $u \in H^{1}(\mathbb{R})$. In the context of (1.1), $\delta$ is interpreted similarly (at each fixed $z$ ). Hence, (1.1) and (1.2) should be understood in the sense of distributions, even though the solutions will be smooth outside of $x=0$.

For the sake of brevity, in what follows below we call these localized bound states 'solitons'. Originally, only solitary waves in integrable systems were called solitons, but in current literature this term is used in a loose sense, meaning all kinds of stable self-trapped modes, including those in non-integrable systems.

Problem (1.1)-(1.2) belongs to a family of models featuring the competition between self-focusing cubic and defocusing quintic terms, that have drawn considerable attention in both the physical and the mathematical communities in recent years, see [5]14]16]19]23]30|31] and the references therein. This combination of nonlinearities is well known in optical media, including liquid waveguides [13] and speciality glasses [6]. Especially interesting are colloids containing metallic nanoparticles, where the CQ nonlinearity can be widely adjusted by selecting the radius of the suspended nanoparticles and the colloidal filling factor [12]. Remarkably, the one-dimensional NLS equation with the CQ nonlinearity admits completely stable exact soliton solutions $9[25$, although this equation is not integrable. The exact soliton solutions are available also in the case when both the cubic and quintic terms in the one-dimensional NLS

2000 Mathematics Subject Classification. 35J60; 35B32; 35Q55; 37C75; 74J30; 78A60.

Key words and phrases. nonlinear Schrödinger equation; cubic-quintic nonlinearity; trapping delta potential; bifurcation; stability.

We are grateful to Reika Fukuizumi, Katharina Kienecker and Masaya Maeda for helpful discussions and comments. We also thank the anonymous referee for constructive remarks on the manuscript. F.G. acknowledges the support of the ERC Advanced Grant "Nonlinear studies of water flows with vorticity". B.A.M. acknowledges a partial support from the National Science Center of Poland in the framework of HARMONIA program no. 2012/06/M/ST2/00479. The work of R.M.W. has been supported by the Hertha-Firnberg Program of the FWF, Grant T402-N13. 
equation have the self-focusing sign [24. In the absence of linear potential, the rigorous stability analysis of onedimensional NLS solitons with general double-power nonlinearities can be found in 2022]. Then, the effective linear potential term added to the NLS equation represents a trapping (waveguiding) structure for light beams, induced by an inhomogeneity of the local refractive index. In particular, the delta-function term adequately represents a narrow trap which is able to capture broad solitonic beams.

Existence and stability of bound states of one-dimensional NLS equations with a delta potential and a single powerlaw nonlinearity $|\psi|^{p-1} \psi, p>1$, have been extensively discussed earlier. We refer the reader to [14|15]19|21 for more information about this. From the mathematical point of view, the presence of the delta-function potential has several interesting consequences. The range of values of the propagation constant for solutions in free space (i.e., with $\epsilon=0$ ) is $k \in\left(0, \frac{3}{4}\right)$, and in this case the bifurcation diagram for the bound states is very simple; see Fig. 2 in Section 6 . Namely, the solutions can be parametrized by $k \in\left(0, \frac{3}{4}\right)$, they bifurcate from $u=0$ at $k=0$, their $L^{2}$ norm (i.e., the integral power of the beam in the optical models) is strictly increasing, and diverges as $k \nearrow \frac{3}{4}$. This accounts for the saturation of the nonlinear refractive index for high-power beams in the CQ optical media. The presence of the potential gives rise to a fold bifurcation point located to the right of $k=\frac{3}{4}$. The bifurcating curve now starts off the trivial line at $k=\frac{\epsilon^{2}}{4}$, can be parametrized by $k$ up to $\bar{k}_{\epsilon}=\frac{3}{4}+\frac{\epsilon^{2}}{4}$ where it 'turns backwards', and again blows up in $L^{2}(\mathbb{R})$, but now as $k \searrow \frac{3}{4}$. The respective bifurcation diagrams are displayed in Fig. 35 in Section 6 for various values of the coupling constant $\epsilon>0$. This phenomenon was already observed in [16], where solitons in a cubic focusing-quintic defocusing medium with a square-well potential were studied by means of numerical methods and the variational approximation. In the case of the delta potential considered here, the fold bifurcation can be described by an exact analysis, as demonstrated in Section 3. Since the parametrization by $k$ breaks down at $k=\bar{k}_{\epsilon}$, where the linearization of (1.2) becomes singular, we resort to a result of Crandall and Rabinowitz [1] which provides a natural framework to deal with this situation.

An important remark at this stage is the multiplicity of positive solutions of (1.2) for $k \in\left(\frac{3}{4}, \bar{k}_{\epsilon}\right)$. In fact, the first step of our analysis, in Section 2 is the explicit determination of all positive solutions of (1.2), in terms of elementary functions; this is a noteworthy feature of the present model. Of course, the expressions obtained are somewhat cumbersome, yet we are able to extract important information from them, notably as regards the stability of the bound states of (1.1). We will thus show explicitly that, for each fixed $k \in\left(\frac{3}{4}, \bar{k}_{\epsilon}\right)$, there are exactly two positive solutions of (1.2), and that the corresponding bound states of (1.1) are both stable. This bistability phenomenon was previously observed numerically in [16 for the square-well potential; see also 31 . In the present context, we can prove the stability rigorously. The fact that the 'upper branch' is stable, while the $L^{2}$ norm of the solutions is decreasing along it, appeared puzzling when it was first discovered in [16. However, in the case of a delta potential considered here, a careful analysis reveals that the spectrum of the linearization of (1.2) is strictly positive along the upper branch, and its stability then follows from the general theory of orbital stability in [17. Along the 'lower branch', the linearized operator has one simple negative eigenvalue, and the rest of its spectrum is positive. In this case, the Vakhitov-Kolokolov (VK) stability criterion [29] (which requires the $L^{2}$ norm to be increasing in $k$ ) ensures stability. Note that, for each fixed $k \in\left(\frac{\epsilon^{2}}{4}, \frac{3}{4}\right)$, the positive solution of (1.2) is unique, and the corresponding bound state is also stable. Therefore, all positive solutions of (1.2) give rise to stable bound states of (1.1). The stability analysis is carried out in full detail in Section 5

It is noteworthy that there is no stability swap at the fold bifurcation point, in sharp contrast with the usual picture in finite-dimensional dynamical systems. Moreover, the bistability of coexisting bound states with different powers and same propagation constant offers potential applications to optics in terms of switching and other elements of all-optical data processing [16].

We would also like to comment on the important role symbolic computer calculations (using Mathematica) and numerical simulations played in our analysis. Mathematica was a powerful tool to compute exact formulas that were too involved to be dealt with manually. This transpires both in the calculation of solutions in the regime $k \in\left(\frac{3}{4}, \bar{k}_{\epsilon}\right)$ in Section 2, and in the stability analysis of Section [5 On the other hand, numerical experiments were very useful at early stages of this work, in order to understand the behavior of solutions, before their explicit representations had been found. We used the so-called 'continuous normalized gradient flow' (CNGF), which was studied and implemented 
in [2] in the context of the NLS equation with a cubic nonlinearity. The excellent agreement between the numerical and the exact solutions (see Fig. 6 8) demonstrates the effectiveness of this scheme in the context of (1.2). The CNGF method being based on constraint minimization (see Section 6), this also suggests that the positive solutions of (1.2) should admit a variational characterization. Our analytical approach allows us to describe the spectral and stability properties of the bound states of (1.1) without resorting to such a characterization. This would however present an interest on its own; see for instance 14 for results in this direction in the case of a delta potential combined with a single power nonlinearity.

Lastly, it is relevant to mention that recent numerical and analytical considerations have demonstrated that the same delta-like attractive potential may effectively stabilize trapped solitons in the NLS equation with a combination of defocusing cubic and focusing quintic terms (the signs opposite to those dealt with in the present work) [32. In the free-space version of the latter equation, all solitons are completely unstable.

Note that, in the present work, we have decided to focus on the case of an attractive delta potential, i.e., $\epsilon>0$. Even though this would deserve a rigorous proof, the physical intuition is that the repulsive case $(\epsilon<0)$ yields unstable solitons, which will tend to escape from the trapped state, to the left or to the right of $x=0$. We conjecture that, in this case, the $H^{1}$ solutions are unstable under general $H^{1}$ perturbations, but remain stable under radial perturbations, as in the case of a single-power nonlinearity studied in [14]19.

\section{Explicit SOLUtions}

We first establish some elementary properties of $H^{1}$ solutions of (1.2). In particular they are all positive. Signchanging solutions exist in the form of cnoidal waves pinned to the delta potential, but those are periodic solutions, not localized ones.

Proposition 1. Let $k>0$ and $u \in H^{1}(\mathbb{R})$ be a non-trivial solution of (1.2). Then u satisfies:

(i) $u^{\prime \prime}-k u+2 u^{3}-u^{5}=0, x \neq 0$;

(ii) $\pm u>0$ on $\mathbb{R}$;

(iii) $u$ is even on $\mathbb{R}$;

(iv) $u \in C^{2}(\mathbb{R} \backslash\{0\}) \cap C(\mathbb{R})$;

(v) $u^{\prime}\left(0^{ \pm}\right)=\mp(\epsilon / 2) u(0)$;

(vi) $u(x), u^{\prime}(x) \rightarrow 0$ as $|x| \rightarrow \infty$.

Proof. Properties (i) and (iv) follow by a standard bootstrap argument using test functions in $C_{0}^{\infty}(\mathbb{R} \backslash\{0\}$ ) (see for instance [7, Section 8]). This argument in fact yields $u \in H^{2}(\mathbb{R} \backslash\{0\})$, from which (vi) follows.

For (ii), first observe that, if $u$ is a solution, so is $-u$. Now suppose by contradiction that there exists $x_{0}>0$ such that $u\left(x_{0}\right)=0$ (the case $x_{0}<0$ is handled similarly). If $u^{\prime}\left(x_{0}\right)=0$, Cauchy's uniqueness theorem implies $u \equiv 0$ on $(0, \infty)$. Since $u$ is continuous, (2.1) below then implies $u \equiv 0$ on $\mathbb{R}$. Suppose now that $u^{\prime}\left(x_{0}\right) \neq 0$. Multiplying the equation by $u^{\prime}$ and integrating from $x_{0}$ to $x>0$ yields

$$
\left(u^{\prime}\right)^{2}(x)-k u^{2}(x)+u^{4}(x)-\frac{1}{3} u^{6}(x)=\left(u^{\prime}\right)^{2}\left(x_{0}\right)>0 \quad \text { for all } x>0 .
$$

However, integrating from $x$ to $+\infty$ and using $u \in H^{2}(\mathbb{R} \backslash\{0\})$ yields

$$
\left(u^{\prime}\right)^{2}(x)-k u^{2}(x)+u^{4}(x)-\frac{1}{3} u^{6}(x)=0 \quad \text { for all } x>0 .
$$

This contradiction shows that $\pm u>0$ on $\mathbb{R}$.

To prove (v), one first establishes that

$$
u^{\prime}\left(0^{+}\right)-u^{\prime}\left(0^{-}\right)=-\epsilon u(0)
$$

by integrating (1.2) over $[-t, t]$ and letting $t \rightarrow 0^{+}$. Then, multiplying (1.2) by $u^{\prime}$ and integrating from $0^{+}$to $+\infty$ yields

$$
\left(u^{\prime}\right)^{2}\left(0^{+}\right)=k u^{2}(0)-u^{4}(0)+\frac{1}{3} u^{6}(0) .
$$


Similarly, integrating from $-\infty$ to $0^{-}$yields

$$
\left(u^{\prime}\right)^{2}\left(0^{-}\right)=k u^{2}(0)-u^{4}(0)+\frac{1}{3} u^{6}(0),
$$

so that $\left|u^{\prime}\left(0^{+}\right)\right|=\left|u^{\prime}\left(0^{-}\right)\right|$. Now, if $u^{\prime}\left(0^{+}\right)=u^{\prime}\left(0^{-}\right)$then $u(0)=0$ by (2.1). If $u^{\prime}\left(0^{+}\right)=u^{\prime}\left(0^{-}\right)=0$ then Cauchy's theorem implies $u \equiv 0$. On the other hand, if $u^{\prime}\left(0^{+}\right)=u^{\prime}\left(0^{-}\right) \neq 0$ then $u$ becomes negative close to $x=0$, a contradiction. Therefore, $u^{\prime}\left(0^{+}\right)=-u^{\prime}\left(0^{-}\right)$and (iv) follows from (2.1).

Finally, (iii) follows by observing that $w(x):=u(x)-u(-x)$ satisfies the initial value problem

$$
w^{\prime \prime}(x)-k w(x)+2 a(x) w(x)-b(x) w(x)=0, \quad w(0)=w^{\prime}(0)=0,
$$

where

$$
a(x)=u^{2}(x)+u(x) u(-x)+u^{2}(-x), \quad b(x)=u^{4}(x)+u^{3}(x) u(-x)+u^{2}(x) u^{2}(-x)+u(x) u^{3}(-x)+u^{4}(-x) .
$$

Cauchy's theorem then implies $w \equiv 0$.

We shall henceforth focus on positive solutions. We will show that they can all be expressed in terms of elementary functions, which is a remarkable feature of the present model. This is especially striking in the range of the propagation constant $k>3 / 4$ which is not allowed in free space (i.e., when $\epsilon=0)$ 925.

First, multiplying (1.2) by $u^{\prime}$ and integrating from $x>0$ to $\infty$, respectively from $-\infty$ to $x<0$, we get

$$
\left(u^{\prime}\right)^{2}(x)-k u^{2}(x)+u^{4}(x)-(1 / 3) u^{6}(x)=0, \quad x \neq 0 .
$$

In particular, taking the limit $x \rightarrow 0^{ \pm}$and using Proposition 1 (v) yields, assuming that $u(0) \neq 0$,

$$
u^{4}(0)-3 u^{2}(0)+3\left(k-\epsilon^{2} / 4\right)=0,
$$

the solutions of which are

$$
u_{ \pm, k, \epsilon}^{2}(0)=\frac{3}{2}\left(1 \pm \sqrt{1-\frac{4}{3}\left(k-\frac{\epsilon^{2}}{4}\right)}\right) .
$$

Note that both $u_{ \pm, k, \epsilon}^{2}(0)$ exist and are positive if and only if

$$
\frac{\epsilon^{2}}{4}<k \leqslant \frac{3}{4}+\frac{\epsilon^{2}}{4}
$$

Next, with a view of further integrating (2.2), we express $u^{\prime}$ as

$$
u^{\prime}(x)= \pm u(x) \sqrt{\frac{1}{3} u^{4}(x)-u^{2}(x)+k}, \quad x \neq 0
$$

(recall we seek solutions with $u>0$ ). The positivity condition for (2.6) to hold reads $u^{2}(x) \in\left(0, \tilde{u}_{-}^{2}\right) \cup\left(\tilde{u}_{+}^{2}, \infty\right)$, where

$$
\tilde{u}_{ \pm}^{2}=\frac{3}{2}\left(1 \pm \sqrt{1-\frac{4 k}{3}}\right) \text {. }
$$

Since positive solutions satisfying (2.6) are even and strictly decreasing in $x>0$, the continuity and the decay of $u$ at infinity only allow for]

$$
u^{2}(0) \leqslant \tilde{u}_{-}^{2}=\frac{3}{2}\left(1-\sqrt{1-\frac{4 k}{3}}\right), \quad \frac{\epsilon^{2}}{4}<k<\frac{3}{4} .
$$

If $k>3 / 4$, (2.6) is well defined without further restriction on $u(0)$, and condition (2.8) is void. (The nature of the degeneracy at $k=3 / 4$ will become more apparent later.) In view of (2.4), (2.5) and (2.8), we identify two different regimes (see the bifurcation diagrams for various values of $\epsilon$ in Section 6 ):

(A) $\frac{\epsilon^{2}}{4}<k<\frac{3}{4}$ : there is only one soliton, $u_{-, k, \epsilon}$, corresponding to $u_{-, k, \epsilon}^{2}(0)$;

(B) $\frac{3}{4}<k<\frac{3}{4}+\frac{\epsilon^{2}}{4}$ : there are two different solitons, $u_{ \pm, k, \epsilon}$, corresponding respectively to $u_{ \pm, k, \epsilon}^{2}(0)$.

\footnotetext{
${ }^{1}$ Note that $\tilde{u}_{-}^{2}=\psi_{\text {sol }}(0,0)^{2}$ in $[5]$.
} 
Notice, in particular, that regime (A) is void if $\epsilon \geqslant \sqrt{3}$; so we will suppose $0<\epsilon<\sqrt{3}$ from now on. Also, we already see from the above analysis that a fold bifurcation occurs at $\bar{k}_{\epsilon}=\frac{3}{4}+\frac{\epsilon^{2}}{4}$ where two distinct solutions merge and disappear (there is no soliton for $k>\bar{k}_{\epsilon}$ ).

From (2.6) and the previous discussion, any positive solution of (1.2) with $\frac{\epsilon^{2}}{4}<k<\frac{3}{4}+\frac{\epsilon^{2}}{4}$ decaying at infinity satisfies

$$
u^{\prime}(x)=-\operatorname{sgn}(x) u(x) \sqrt{\frac{1}{3} u^{4}(x)-u^{2}(x)+k}, \quad x \neq 0 .
$$

In particular, $u$ is even, $u^{\prime}(x)<0$ for $x>0$, and $\lim _{x \rightarrow \infty} u^{\prime}(x) / u(x)=-\sqrt{k}$, so $u(x)$ decays like $\mathrm{e}^{-\sqrt{k}|x|}$ as $|x| \rightarrow \infty$.

Now (2.9) is a first order ODE with separated variables, which can be integrated explicitly. Alternatively, the solutions in regime (A) are easily constructed by applying some surgery to the known explicit solitons in free space, given in 925 as

$$
u_{-, k, 0}(x)=\sqrt{\frac{2 k}{1+\sqrt{1-\frac{4 k}{3}} \cosh (2 \sqrt{k} x)}}, \quad 0<k<\frac{3}{4} .
$$

The corresponding solutions pinned to the delta potential with $\epsilon>0$ are obtained as

$$
u_{-, k, \epsilon}(x)=\sqrt{\frac{2 k}{1+\sqrt{1-\frac{4 k}{3}} \cosh (2 \sqrt{k}(|x|+\xi))}}, \quad \frac{\epsilon^{2}}{4}<k<\frac{3}{4},
$$

where $\xi=\xi(k, \epsilon)$ is determined by the jump condition in Proposition 1 (v), which yields

$$
\frac{\sinh (2 \sqrt{k} \xi)}{1+\sqrt{1-\frac{4 k}{3}} \cosh (2 \sqrt{k} \xi)}=\frac{\epsilon}{2 \sqrt{k}} \frac{1}{\sqrt{1-\frac{4 k}{3}}} .
$$

It is not difficult to check that this equation has a unique solution $\xi \in \mathbb{R}$ if $k>\frac{\epsilon^{2}}{4}$. In fact this solution can be computed explicitly:

$$
\mathrm{e}^{2 \sqrt{k} \xi}=\frac{\epsilon+\epsilon \sqrt{1+\left(\frac{4 k}{\epsilon^{2}}-1\right)\left(1-\frac{4 k}{3}\right)}}{2\left(\sqrt{k}-\frac{\epsilon}{2}\right) \sqrt{1-\frac{4 k}{3}}} .
$$

Thus, the solutions in (2.11) take the form of

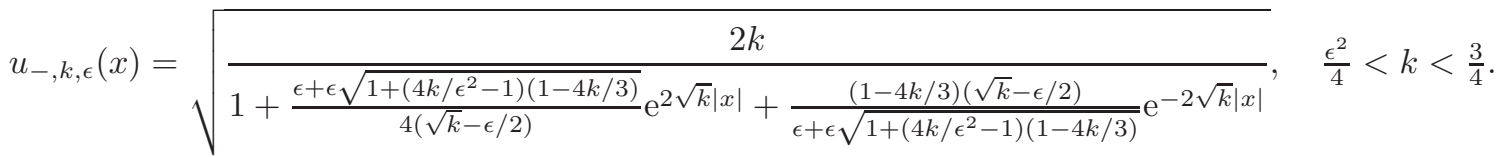

For $k=3 / 4$, a similar procedure applied to the 'front soliton' given in Eq. (11) of [5] yields a solution

$$
u_{f, \epsilon}(x)=\sqrt{\frac{3}{2}}\left[1+\frac{\epsilon}{\sqrt{3}-\epsilon} \mathrm{e}^{\sqrt{3}|x|}\right]^{-1 / 2} .
$$

As can be seen in Section 6 by comparing the bifurcation diagrams for solutions in free space to those with $\epsilon>0$, solutions with $k>3 / 4$ only exist in the presence of the potential. In other words, regime (B) above is void for $\epsilon=0$. Therefore, no free-space solutions are available that could be pinned to the delta potential by the same sort of surgery as above, and one has to integrate the equation manually. We integrate (2.9) using an Euler substitution, which yields

$$
u_{ \pm, k, \epsilon}(x)=2 \sqrt{\frac{k}{\left(\mathrm{e}^{\sqrt{k}(|x|-c)}+\mathrm{e}^{-\sqrt{k}(|x|-c)}\right)\left(\left(2 \sqrt{\frac{k}{3}}+1\right) \mathrm{e}^{\sqrt{k}(|x|-c)}-\left(2 \sqrt{\frac{k}{3}}-1\right) \mathrm{e}^{-\sqrt{k}(|x|-c)}\right)}}, \quad \frac{3}{4}<k<\frac{3}{4}+\frac{\epsilon^{2}}{4},
$$


where the integration constant $c=c_{ \pm, k, \epsilon} \in \mathbb{R}$ can be determined from (2.4). The expressions for the integration constants are somewhat cumbersome. They can be computed using Mathematica, which yield:2

$$
\mathrm{e}^{\sqrt{k} c_{-, k, \epsilon}}=\sqrt{\frac{3-\sqrt{3} \sqrt{3+\epsilon^{2}-4 k}+2 \epsilon \sqrt{k}-4 k}{-3+\sqrt{3} \sqrt{3+\epsilon^{2}-4 k}+2 \sqrt{3} \sqrt{k}-2 \sqrt{k} \sqrt{3+\epsilon^{2}-4 k}}}
$$

and

$$
\mathrm{e}^{\sqrt{k} c_{+, k, \epsilon}}=\sqrt{\frac{-3-\sqrt{3} \sqrt{3+\epsilon^{2}-4 k}-2 \epsilon \sqrt{k}+4 k}{3+\sqrt{3} \sqrt{3+\epsilon^{2}-4 k}-2 \sqrt{3} \sqrt{k}-2 \sqrt{k} \sqrt{3+\epsilon^{2}-4 k}}} .
$$

Hence the explicit form (2.14) is not very convenient to work with, but we shall see in Section 5 that some information can nevertheless be extracted from it. However, for given values of the parameters, the exact form of the solutions may be useful, especially in numerical calculations. For instance, at the fold bifurcation point, where $\bar{k}_{\epsilon}=\frac{3}{4}+\frac{\epsilon^{2}}{4}$, the solution takes the more tractable form:

$$
\bar{u}_{\epsilon}(x)=\sqrt{\frac{3}{2}} \sqrt{\frac{3+\epsilon^{2}}{3+\epsilon^{2} \cosh \left(\sqrt{3+\epsilon^{2}}|x|\right)+\epsilon \sqrt{3+\epsilon^{2}} \sinh \left(\sqrt{3+\epsilon^{2}}|x|\right)}} .
$$

Remark 1. It can also be checked that, as $\epsilon \rightarrow 0$, the solutions in (2.12) converge to the corresponding free-space solitons in (2.10). It will be seen in the proof of Lemma 2 that, in fact, they can be extended to a holomorphic family of functions parametrized by $\epsilon$ in a complex domain containing zero.

\section{The Bifurcation AnALYsis}

In this section we will embed the above explicit solutions in a bifurcation-theoretic framework, suitable to the rigorous stability analysis which will be carried out in Section 5. We will prove the following result.

Theorem 1. Let $\epsilon \in(0, \sqrt{3})$. The solutions $(k, u)$ of (1.2) obtained in (2.12)-(2.14) form a smooth curve in $\mathbb{R} \times H^{1}(\mathbb{R})$, which bifurcates from the trivial solution $u \equiv 0$ at $k=\frac{\epsilon^{2}}{4}$, consists of the solutions $u_{-, k, \epsilon}$ up to $\bar{k}_{\epsilon}=\frac{3}{4}+\frac{\epsilon^{2}}{4}$, where it has a turning point, and then consists of the solutions $u_{+, k, \epsilon}$ and becomes unbounded as $k \searrow 3 / 4$. More precisely,

$$
\lim _{k \searrow \frac{\epsilon^{2}}{4}}\left\|u_{-, k, \epsilon}\right\|_{H^{1}}=0 \quad \text { and } \quad \lim _{k \searrow 3 / 4}\left\|u_{+, k, \epsilon}\right\|_{L^{2}}=\infty .
$$

A good mental picture of Theorem 1 can be grasped from the bifurcation diagrams in Section [6, where $\left\|u_{+, k, \epsilon}\right\|_{L^{2}}$ is plotted against $k$, for various values of the coupling constant $\epsilon>0$.

To prove Theorem 1 first observe that Eq. (1.2) can be formulated as

$$
F_{\epsilon}(k, u)=0,
$$

where

$$
F_{\epsilon}(k, u):=u^{\prime \prime}-k u+\epsilon \delta(x) u+2 u^{3}-u^{5}
$$

can be seen as a mapping $F_{\epsilon}: \mathbb{R} \times H^{1}(\mathbb{R}) \rightarrow H^{-1}(\mathbb{R})$ (by interpreting the right-hand side as a distribution). It is standard to show that this mapping is continuously Fréchet differentiable. The derivative with respect to $u$, which will play a key role in our analysis, is formally given by $D_{u} F_{\epsilon}(k, u): H^{1}(\mathbb{R}) \rightarrow H^{-1}(\mathbb{R})$,

$$
D_{u} F_{\epsilon}(k, u) v=v^{\prime \prime}-k v+\epsilon \delta(x) v+\left[6-5 u^{2}\right] u^{2} v, \quad v \in H^{1}(\mathbb{R}) .
$$

More precisely, following the proof of [19, Lemma 10], $D_{u} F_{\epsilon}(k, u)$ can be interpreted as a self-adjoint operator acting in $L^{2}(\mathbb{R})$, with domain

$$
\mathcal{D}_{\epsilon}=\left\{v \in H^{1}(\mathbb{R}) \cap H^{2}(\mathbb{R} \backslash\{0\}): v^{\prime}\left(0^{+}\right)-v^{\prime}\left(0^{-}\right)=-\epsilon v(0)\right\},
$$

defined by

$$
D_{u} F_{\epsilon}(k, u) v=v^{\prime \prime}-k v+\left[6-5 u^{2}\right] u^{2} v, \quad v \in \mathcal{D}_{\epsilon} .
$$

\footnotetext{
${ }^{2}$ The positivity of the expressions under the square roots can be checked by plotting their graphs (as functions of $\epsilon$ and $k$ ) in Mathematica.
} 
Using the explicit formulas for the solutions obtained in Section 2 (in particular their uniform exponential decay), it can be shown that

$$
\mathcal{S}_{-, \epsilon}=\left\{\left(k, u_{-, k, \epsilon}\right): k \in\left(\frac{\epsilon^{2}}{4}, \bar{k}_{\epsilon}\right)\right\} \quad \text { and } \quad \mathcal{S}_{+, \epsilon}=\left\{\left(k, u_{+, k, \epsilon}\right): k \in\left(\frac{3}{4}, \bar{k}_{\epsilon}\right)\right\}
$$

define two continuous curves in $\mathbb{R} \times H^{1}(\mathbb{R})$. In the remainder of the paper, we will obtain much more information about these sets. It will be convenient to call $\mathcal{S}_{-, \epsilon}$ the lower curve and $\mathcal{S}_{+, \epsilon}$ the upper curve.

Proposition 2. The sets $\mathcal{S}_{\epsilon, \pm}$ are smooth curves of non-degenerate solutions of (3.1), in the sense that $D_{u} F_{\epsilon}(k, u)$ is non-singular along $\mathcal{S}_{-, \epsilon}$ and $\mathcal{S}_{+, \epsilon}$. Furthermore, $\mathcal{S}_{-, \epsilon}$ bifurcates from the point $\left(\frac{\epsilon^{2}}{4}, 0\right)$ in $\mathbb{R} \times H^{1}(\mathbb{R})$, and meets $\mathcal{S}_{+, \epsilon}$ at the point $\left(\bar{k}_{\epsilon}, \bar{u}_{\epsilon}\right)$, where $D_{u} F_{\epsilon}(k, u)$ becomes singular.

Proof. First, it is easily seen that

$$
\operatorname{ker} D_{u} F_{\epsilon}\left(\frac{\epsilon^{2}}{4}, 0\right)=\operatorname{span}\left\{\mathrm{e}^{-\frac{\epsilon}{2}|x|}\right\}
$$

so that zero is a simple eigenvalue of $D_{u} F_{\epsilon}\left(\frac{\epsilon^{2}}{4}, 0\right)$. It then follows from standard bifurcation theory that $\mathcal{S}_{-, \epsilon}$ bifurcates from $\left(\frac{\epsilon^{2}}{4}, 0\right)$. More precisely, the Crandall-Rabinowitz theorem [10, Theorem 1.7] yields the existence of a unique local continuous curve of solutions bifurcating from the line of trivial solutions $\{(k, 0): k \in \mathbb{R}\}$ in $\mathbb{R} \times H^{1}(\mathbb{R})$ at the point $\left(\frac{\epsilon^{2}}{4}, 0\right)$. Since our explicit solutions all belong to $H^{1}(\mathbb{R})$, they coincide with the Crandall-Rabinowitz curve in a neighborhood of $\left(\frac{\epsilon^{2}}{4}, 0\right)$ in $\mathbb{R} \times H^{1}(\mathbb{R})$.

The smoothness of the curves $\mathcal{S}_{-, \epsilon}$ and $\mathcal{S}_{+, \epsilon}$ follows from the implicit function theorem in $\mathbb{R} \times H^{1}(\mathbb{R})$, provided that $D_{u} F_{\epsilon}(k, u): H^{1}(\mathbb{R}) \rightarrow H^{-1}(\mathbb{R})$ is non-singular along the solution curves, which is given by Lemma 1 below.

Remark 2. Note that the global bifurcation theorem of Rabinowitz [26, Theorem 1.3] could be applied here to deduce the existence of a connected set of solutions, bifurcating from the point $\left(\frac{\epsilon^{2}}{4}, 0\right)$, where we have only shown that local bifurcation occurs. But our proof is more straightforward — we construct the curve using the explicit solutions we found in Section 2 - and provides a smooth curve, which is stronger than the conclusion of Rabinowitz's theorem. We shall use Rabinowitz's theorem to infer that the bifurcating curve is unbounded (see the conclusion of the proof of Theorem 1 below). But this can also be deduced from the explicit form of the solutions (see Eq. (5.8) below).

In view of the more detailed spectral analysis that will be carried out later, and in order to follow the usual sign convention of the spectral theory of Schrödinger operators, it is convenient to introduce the self-adjoint operators $T_{ \pm, k, \epsilon}: \mathcal{D}_{\epsilon} \subset L^{2}(\mathbb{R}) \rightarrow L^{2}(\mathbb{R})$,

$$
T_{ \pm, k, \epsilon} v:=-D_{u} F_{\epsilon}\left(k, u_{ \pm, k, \epsilon}\right) v=-v^{\prime \prime}+k v-\left[6-5 u_{ \pm, k, \epsilon}^{2}(x)\right] u_{ \pm, k, \epsilon}^{2}(x) v
$$

Formally, one can write

$$
T_{ \pm, k, \epsilon} v=-v^{\prime \prime}+k v-\epsilon \delta(x) v-\left[6-5 u_{ \pm, k, \epsilon}^{2}(x)\right] u_{ \pm, k, \epsilon}^{2}(x) v .
$$

Thus, $T_{ \pm, k, \epsilon}$ can be seen as an operator acting between $H^{1}(\mathbb{R})$ and $H^{-1}(\mathbb{R})$, by interpreting the right-hand side as a distribution.

The proof of the next lemma relies on the spectral theory of $-\frac{\mathrm{d}^{2}}{\mathrm{~d} x^{2}}+k: \mathcal{D}_{\epsilon} \subset L^{2}(\mathbb{R}) \rightarrow L^{2}(\mathbb{R})$, which has been formalized in the classic book [1].

Lemma 1. The linearized operator (3) satisfies:

(i) $T_{-, k, \epsilon}: H^{1}(\mathbb{R}) \rightarrow H^{-1}(\mathbb{R})$ is an isomorphism for all $k \in\left(\frac{\epsilon^{2}}{4}, \bar{k}_{\epsilon}\right)$;

(ii) $T_{+, k, \epsilon}: H^{1}(\mathbb{R}) \rightarrow H^{-1}(\mathbb{R})$ is an isomorphism for all $k \in\left(\frac{3}{4}, \bar{k}_{\epsilon}\right)$;

(iii) $D_{u} F_{\epsilon}\left(\bar{k}_{\epsilon}, \bar{u}_{\epsilon}\right)$ is singular with

$$
\operatorname{ker} D_{u} F_{\epsilon}\left(\bar{k}_{\epsilon}, \bar{u}_{\epsilon}\right)=\operatorname{span}\left\{\eta_{\epsilon}\right\}, \quad \eta_{\epsilon}=\left|\bar{u}_{\epsilon}^{\prime}\right| .
$$

Furthermore, since $\eta_{\epsilon}>0$, zero is the principal eigenvalue of $D_{u} F_{\epsilon}\left(\bar{k}_{\epsilon}, \bar{u}_{\epsilon}\right)$. 
Proof. A first important remark is that each operator $T_{ \pm, k, \epsilon}$ is a compact perturbation of $-\frac{\mathrm{d}^{2}}{\mathrm{~d} x^{2}}+k: H^{1}(\mathbb{R}) \rightarrow H^{-1}(\mathbb{R})$, the latter being an isomorphism for all $k>0$. It then follows from standard spectral theory (see [11827) that the spectrum of $T_{ \pm, k, \epsilon}: \mathcal{D}_{\epsilon} \subset L^{2}(\mathbb{R}) \rightarrow L^{2}(\mathbb{R})$ consists of a finite number of isolated simple eigenvalues lying below a continuous part $[k, \infty)$. Furthermore, $T_{ \pm, k, \epsilon}: H^{1}(\mathbb{R}) \rightarrow H^{-1}(\mathbb{R})$ is an isomorphism if and only if

$$
\operatorname{ker} T_{ \pm, k, \epsilon}=\{0\} .
$$

We will now show that (3.5) holds for all $\frac{\epsilon^{2}}{4}<k<\bar{k}_{\epsilon}$. If $T_{ \pm, k, \epsilon} v=0$ then $v \in H^{1}(\mathbb{R}) \cap H^{2}(\mathbb{R} \backslash\{0\})$ satisfies

$$
\begin{gathered}
-v^{\prime \prime}+k v-\left[6-5 u_{ \pm, k, \epsilon}^{2}(x)\right] u_{ \pm, k, \epsilon}^{2}(x) v=0, \quad x \neq 0, \\
v^{\prime}\left(0^{+}\right)-v^{\prime}\left(0^{-}\right)=-\epsilon v(0) .
\end{gathered}
$$

Applying Theorem 3.3 of $[3$ to (3.6) separately on $(-\infty, 0)$ and $(0, \infty)$, and using the continuity of $v$, there exists a constant $\alpha \in \mathbb{R}$ such that $v=\alpha\left|u_{ \pm, k, \epsilon}^{\prime}\right|$. Hence,

$$
v(0)=-\alpha u_{ \pm, k, \epsilon}^{\prime}\left(0^{-}\right)=\alpha u_{ \pm, k, \epsilon}^{\prime}\left(0^{+}\right)=-\alpha \frac{\epsilon}{2} u_{ \pm, k, \epsilon}(0),
$$

and since

$$
u_{ \pm, k, \epsilon}^{\prime \prime}\left(0^{-}\right)=u_{ \pm, k, \epsilon}^{\prime \prime}\left(0^{+}\right)=k u_{ \pm, k, \epsilon}(0)-2 u_{ \pm, k, \epsilon}^{3}(0)+u_{ \pm, k, \epsilon}^{5}(0)
$$

it follows from (3.7) that

Combining this with (2.4) yields

$$
4\left[k-2 u_{ \pm, k, \epsilon}^{2}(0)+u_{ \pm, k, \epsilon}^{4}(0)\right]=\epsilon^{2} .
$$

$$
1-\frac{4}{3}\left(k-\frac{\epsilon^{2}}{4}\right)= \pm \sqrt{1-\frac{4}{3}\left(k-\frac{\epsilon^{2}}{4}\right)} .
$$

The ' + ' sign in (3.8) corresponds to $u_{-, k, \epsilon}$ and yields $k=\frac{\epsilon^{2}}{4}$ or $k=\frac{3}{4}+\frac{\epsilon^{2}}{4}$, from which (i) and (iii) follow. The '-' sign in (3.8) corresponds to $u_{+, k, \epsilon}$ and yields $k=\frac{3}{4}+\frac{\epsilon^{2}}{4}$, so (ii) must hold. The lemma is proved.

Even though the linearized operator $D_{u} F_{\epsilon}(k, u)$ becomes singular at $\left(\bar{k}_{\epsilon}, \bar{u}_{\epsilon}\right)$, we have the following result.

Proposition 3. The set

$$
\mathcal{S}:=\mathcal{S}_{-, \epsilon} \cup\left\{\left(\bar{k}_{\epsilon}, \bar{u}_{\epsilon}\right)\right\} \cup \mathcal{S}_{+, \epsilon}
$$

is a smooth curve in $\mathbb{R} \times H^{1}(\mathbb{R})$.

To prove Proposition 3 we will use a theorem of Crandall and Rabinowitz, which enables us to reparametrize the bifurcation curve around the point $\left(\bar{k}_{\epsilon}, \bar{u}_{\epsilon}\right)$, where the parametrization by $k$ breaks down. For the reader's convenience we reproduce this result here.

Theorem 2 (Theorem 3.2 of [11]). Let $\left(k_{0}, u_{0}\right) \in \mathbb{R} \times X$ where $X$ is a Banach space and let $F$ be a continuously differentiable mapping of an open neighborhood of $\left(k_{0}, u_{0}\right)$ into another Banach space $Y$. Suppose that $\operatorname{ker} D_{u} F\left(k_{0}, u_{0}\right)=$ $\operatorname{span}\left\{\eta_{0}\right\}$ is one-dimensional, that codim $\operatorname{rge} D_{u} F\left(k_{0}, u_{0}\right)=1$, and that $D_{k} F\left(k_{0}, u_{0}\right) \notin \operatorname{rge} D_{u} F\left(k_{0}, u_{0}\right)$. If $Z$ is a complement of $\operatorname{span}\left\{\eta_{0}\right\}$ in $X$, then the solutions of $F(k, u)=F\left(k_{0}, u_{0}\right)$ near $\left(k_{0}, u_{0}\right)$ form a curve $(k(s), u(s))=$ $\left(k_{0}+\tau(s), u_{0}+s \eta_{0}+z(s)\right)$, where $s \mapsto(\tau(s), z(s)) \in \mathbb{R} \times Z$ is a continuously differentiable function near $s=0$, and $\tau(0)=\dot{\tau}(0)=0, z(0)=\dot{z}(0)=0$.

Here, the 'dot' denotes differentiation with respect to $s$.

Proof. Apply the implicit function theorem to the function $f: \mathbb{R} \times \mathbb{R} \times Z \rightarrow Y$ defined by

$$
f(s, \tau, z)=F\left(k_{0}+\tau, u_{0}+s \eta_{0}+z\right)
$$

at the point $(s, \tau, z)=(0,0,0)$. 
Proof of Proposition 0. Firstly, since the operator $D_{u} F_{\epsilon}\left(\bar{k}_{\epsilon}, \bar{u}_{\epsilon}\right)$ is self-adjoint, it follows from (3.4) that

$$
\text { codim rge } D_{u} F_{\epsilon}\left(\bar{k}_{\epsilon}, \bar{u}_{\epsilon}\right)=\operatorname{dim} \operatorname{ker} D_{u} F_{\epsilon}\left(\bar{k}_{\epsilon}, \bar{u}_{\epsilon}\right)=1 \text {. }
$$

Furthermore, the range of $D_{u} F_{\epsilon}\left(\bar{k}_{\epsilon}, \bar{u}_{\epsilon}\right)$ is characterized by

$$
\operatorname{rge} D_{u} F_{\epsilon}\left(\bar{k}_{\epsilon}, \bar{u}_{\epsilon}\right)=\left\{v \in L^{2}(\mathbb{R}): \int_{\mathbb{R}} v \eta_{\epsilon} \mathrm{d} x=0\right\} .
$$

Next, we need to check that $D_{k} F_{\epsilon}\left(\bar{k}_{\epsilon}, \bar{u}_{\epsilon}\right) \notin \operatorname{rge} D_{u} F_{\epsilon}\left(\bar{k}_{\epsilon}, \bar{u}_{\epsilon}\right)$. But this is clear, as $D_{k} F_{\epsilon}\left(\bar{k}_{\epsilon}, \bar{u}_{\epsilon}\right)=-\bar{u}_{\epsilon}$ and

$$
\int_{\mathbb{R}} \bar{u}_{\epsilon} \eta_{\epsilon} \mathrm{d} x=2 \int_{0}^{\infty} \bar{u}_{\epsilon} \bar{u}_{\epsilon}^{\prime} \mathrm{d} x=-\bar{u}_{\epsilon}^{2}(0)<0 .
$$

It then follows from Theorem 2 that the solutions of (3.1) in a neighborhood of $\left(\bar{k}_{\epsilon}, \bar{u}_{\epsilon}\right)$ form a smooth curve,

$$
\left\{\left(k_{s}, u_{s}\right): s \in(-\varepsilon, \varepsilon)\right\} \subset \mathbb{R} \times H^{1}(\mathbb{R}) \quad(\text { for some } \varepsilon>0)
$$

such that, at $s=0$,

$$
k_{0}=\bar{k}_{\epsilon}, \dot{k}_{0}=0, \quad u_{0}=\bar{u}_{\epsilon}, \dot{u}_{0}=\eta_{\epsilon} .
$$

Consequently, the lower and upper curves $\mathcal{S}_{-, \epsilon}$ and $\mathcal{S}_{+, \epsilon}$ meet smoothly at the turning point $\left(\bar{k}_{\epsilon}, \bar{u}_{\epsilon}\right)$.

We can now end this section with the

Proof of Theorem 1. In view of Propositions 2 and 3 , we only need to establish the asymptotic behavior of the upper curve as $k \searrow \frac{3}{4}$ to complete the proof of the theorem. But this readily follows from Rabinowitz's global bifurcation theorem [26, Theorem 1.3]. Indeed, this result states the following alternative: either (i) the bifurcating curve meets the trivial line $\{(k, 0): k \in \mathbb{R}\} \subset \mathbb{R} \times H^{1}(\mathbb{R})$ again at a point $\left(k^{*}, 0\right)$ with $k^{*} \neq \frac{\epsilon^{2}}{4}$, or (ii) it is unbounded in $\mathbb{R} \times H^{1}(\mathbb{R})$. In the present context, case (i) is ruled out by the explicit form of the solutions given in Section 2 Therefore, $\mathcal{S}$ is unbounded in $\mathbb{R} \times H^{1}(\mathbb{R})$, and so we must have

$$
\lim _{k \searrow 3 / 4}\left\|u_{+, k, \epsilon}\right\|_{H^{1}}=\infty .
$$

Moreover, by (2.2) and (2.4), there exists a constant $C>0$ (independent of $k$ ) such that

$$
u_{+, k, \epsilon}^{2}(x) \geqslant C\left(u_{+, k, \epsilon}^{\prime}\right)^{2}(x), \quad \text { for all } x \neq 0, k \in\left(\frac{3}{4}, \bar{k}_{\epsilon}\right),
$$

which implies that $\lim _{k \searrow 3 / 4}\left\|u_{+, k, \epsilon}\right\|_{H^{1}}=\infty$ if and only if

$$
\lim _{k \searrow 3 / 4}\left\|u_{+, k, \epsilon}\right\|_{L^{2}}=\infty
$$

and concludes the proof of Theorem 1

\section{Spectral PROperties}

The purpose of this section is to prove the following spectral result, which is a first step towards our stability theorem. Let $n\left(T_{ \pm, k, \epsilon}\right)$ denote the number of negative eigenvalues of the self-adjoint operator $T_{ \pm, k, \epsilon}$.

Proposition 4. The spectrum of the linear operator $T_{ \pm, k, \epsilon}: \mathcal{D}_{\epsilon} \subset L^{2}(\mathbb{R}) \rightarrow L^{2}(\mathbb{R})$ consists of a finite number of simple isolated eigenvalues and a continuous part $[k, \infty)$. Furthermore,

$$
n\left(T_{-, k, \epsilon}\right)=1 \quad \text { for all } k \in\left(\frac{\epsilon^{2}}{4}, \bar{k}_{\epsilon}\right)
$$

and

$$
n\left(T_{+, k, \epsilon}\right)=0 \quad \text { for all } k \in\left(\frac{3}{4}, \bar{k}_{\epsilon}\right) .
$$


Proof. As noted earlier in the proof of Lemma 1, the basic structure of the spectrum of $T_{ \pm, k, \epsilon}$ follows from standard spectral theory, see for instance 11827. For the simplicity of eigenvalues, suppose that $u, v \in \mathcal{D}_{\epsilon}$ are eigenfunctions of $T_{ \pm, k, \epsilon}$ corresponding to an eigenvalue $\lambda<k$. Then $\left(u v^{\prime}-u^{\prime} v\right)^{\prime}=u v^{\prime \prime}-u^{\prime \prime} v=0$ on $\mathbb{R} \backslash\{0\}$, therefore there exists a constant $C \in \mathbb{R}$ such that $u v^{\prime}-u^{\prime} v=C$ on $\mathbb{R} \backslash\{0\}$. However, $\lim _{|x| \rightarrow \infty} u v^{\prime}-u^{\prime} v=0$, as $u, v \in H^{2}(\mathbb{R} \backslash\{0\})$. Hence $C=0$ and $u, v$ are linearly dependent.

Next, (4.1) follows from a perturbation analysis similar to the proof of Lemma 12 in [19. The idea is first to observe that the property holds when $\epsilon=0$. Indeed, in this case the kernel of the linearization at the free-space soliton (2.10) is spanned by its derivative $u_{-, k, 0}^{\prime}$, which has a unique zero at $x=0$, where it changes sign. Therefore, by the Sturm's oscillation theorem, zero is the second eigenvalue of the linearization, and so $n\left(T_{-, k, 0}\right)=1$. With this information at hand, we then use perturbation theory to make sure that the second eigenvalue becomes positive for small values of $\epsilon>0$. A first step in this direction is the smoothness of the family of operators $T_{-, k, \epsilon}$ at $\epsilon=0$.

Lemma 2. For any $\rho>0$ small enough, there exists a open connected neighborhood $\Omega$ of the real-line segment $[0,2 \sqrt{k}-\rho]$ in $\mathbb{C}$, such that $\left\{T_{-, k, \epsilon}: \epsilon \in \Omega\right\}$ is a holomorphic family of operators.

Proof. The result follows in a similar way to Lemma 13 in [19, and is based on the notion of holomorphic family of unbounded operators of type (B) in the sense of Kato, see Theorem 4.2 in chapter VII of [18. The argument boils down to checking that, for each fixed $x \in \mathbb{R}$, the mapping $\epsilon \mapsto u_{-, k, \epsilon}(x)$ is holomorphic on a suitable domain, independent of $x$. That this domain can be taken as stated in Lemma 2 follows by a careful inspection of (2.12), and the observation that the function $\epsilon \mapsto \sqrt{\epsilon^{2}+\left(4 k-\epsilon^{2}\right)(1-4 k / 3)}$ is holomorphic on the strip $\{\epsilon \in \mathbb{C}: \operatorname{Im} \epsilon \in$ $(-\sqrt{3-4 k}, \sqrt{3-4 k})\}$.

Thanks to Lemma 2] standard perturbation theory [18 yields two holomorphic mappings,

$$
\Omega \ni \epsilon \mapsto \lambda_{\epsilon} \in \mathbb{R}, \quad \Omega \ni \epsilon \mapsto w_{\epsilon} \in \mathcal{D}_{\epsilon}
$$

such that $\lambda_{0}=0, w_{0}=u_{-, k, 0}^{\prime}$, where $\lambda_{\epsilon}$ and $w_{\epsilon}$ are, respectively, the second eigenvalue and eigenvector of $T_{-, k, \epsilon}$ :

$$
T_{-, k, \epsilon} w_{\epsilon}=\lambda_{\epsilon} w_{\epsilon}, \quad \epsilon \in \Omega .
$$

We will now show that $\dot{\lambda}_{0}>0$, which implies that the second eigenvalue of $T_{-, k, \epsilon}$ is positive for small $\epsilon>0$. We use the 'dot' here to denote differentiation with respect to $\epsilon, \dot{\lambda}_{0}>0$ being the derivative of $\lambda_{\epsilon}$ with respect to $\epsilon$ at $\epsilon=0$, and similarly for other quantities below. Differentiating (4.3) with respect to $\epsilon$ at $\epsilon=0$ yields

$$
-\dot{w}_{0}^{\prime \prime}+k \dot{w}_{0}-4\left[3-5 u_{0}^{2}\right] u_{0} \dot{u}_{0} u_{0}^{\prime}-\left[6-5 u_{0}^{2}\right] u_{0}^{2} \dot{w}_{0}=\dot{\lambda}_{0} \dot{w}_{0},
$$

where we have put $u_{0} \equiv u_{-, k, 0}$ and $\dot{u}_{0} \equiv \dot{u}_{-, k, 0}$ to simplify the notation. Observe that $w_{\epsilon} \in \mathcal{D}_{\epsilon} \Rightarrow \dot{w}_{0} \in \mathcal{D}_{0}=H^{2}(\mathbb{R})$. Now, differentiating (1.2) with respect to $\epsilon$ at $\epsilon=0$ shows that

$$
T_{0} \dot{u}_{0}:=-\dot{u}_{0}^{\prime \prime}+k \dot{u}_{0}-\left[6-5 u_{0}^{2}\right] u_{0}^{2} \dot{u}_{0}=\delta(x) u_{0} .
$$

Multiplying (4.4) by $u_{0}^{\prime}$, integrating by parts and using $T_{0} u_{0}^{\prime}=0$ then yields

$$
\dot{\lambda}_{0}=\frac{4 \int_{\mathbb{R}}\left[5 u_{0}^{2}-3\right] u_{0} \dot{u}_{0}\left(u_{0}^{\prime}\right)^{2}}{\int_{\mathbb{R}}\left(u_{0}^{\prime}\right)^{2}} .
$$

Furthermore, straightforward calculations show that

$$
4\left[5 u_{0}^{2}-3\right] u_{0}\left(u_{0}^{\prime}\right)^{2}=T_{0}\left(-k u_{0}+2 u_{0}^{3}-u_{0}^{5}\right)=T_{0}\left(-u_{0}^{\prime \prime}\right),
$$

and it follows by (4.5) that

$$
4 \int_{\mathbb{R}}\left[5 u_{0}^{2}-3\right] u_{0}\left(u_{0}^{\prime}\right)^{2} \dot{u}_{0}=\left(T_{0}\left(-u_{0}^{\prime \prime}\right), \dot{u}_{0}\right)_{L^{2}}=\left(-u_{0}^{\prime \prime}, T_{0} \dot{u}_{0}\right)_{L^{2}}=-u_{0}(0)^{\prime \prime} u(0)>0,
$$

showing that $\dot{\lambda}_{0}$ is indeed positive. This implies that (4.1) holds for $\epsilon>0$ small enough. To complete the proof of (4.1), we invoke the continuous dependence of the first two eigenvalues of $T_{-, k, \epsilon}$ on $\epsilon \in[0,2 \sqrt{k}$ ) (given by Lemma 2), and the fact that the eigenvalues cannot cross zero unless $\epsilon=2 \sqrt{k}$ (Lemma 1 (i)). 
We now turn to the proof of (4.2). By (4.1), the first eigenvalue of $T_{-, k, \epsilon}$ is negative, for all $k \in\left(\frac{\epsilon^{2}}{4}, \bar{k}_{\epsilon}\right)$. Since $\operatorname{ker} T_{ \pm, k, \epsilon} \neq\{0\} \Leftrightarrow k=\bar{k}_{\epsilon}$ by Lemma1, we only need to show that the first eigenvalue of $T_{ \pm, k, \epsilon}$ crosses zero at $\left(\bar{k}_{\epsilon}, \bar{u}_{\epsilon}\right)$. Using the parametrization (3.10), and denoting by $\mu_{s}$ the first eigenvalue along the curve, this amounts to showing that $\dot{\mu}_{0} \neq 0$. The first eigenvalue and eigenfunction $\mu_{s}, v_{s}$ satisfy $\mu_{0}=0, v_{0}=\eta_{\epsilon}$, and

$$
v_{s} \in \mathcal{D}_{\epsilon}, \quad-v_{s}^{\prime \prime}+k_{s} v_{s}-\left[6 u_{s}^{2}-5 u_{s}^{4}\right] v_{s}=\mu_{s} v_{s}, \quad s \in(-\varepsilon, \varepsilon) .
$$

In view of (3.11), differentiating with respect to $s$ and letting $s=0$ yields

$$
-\dot{v}_{0}^{\prime \prime}+\bar{k}_{\epsilon} \dot{v}_{0}-4\left[3-5 \bar{u}_{\epsilon}^{2}\right] \bar{u}_{\epsilon} \eta_{\epsilon}^{2}-\left[6-5 \bar{u}_{\epsilon}^{2}\right] \bar{u}_{\epsilon}^{2} \dot{v}_{0}=\dot{\mu}_{0} \eta_{\epsilon},
$$

where the 'dot' now denotes again differentiation with respect to $s$. Multiplying both sides of (4.7) by $\eta_{\epsilon}$, integrating by parts and using $D_{u} F_{\epsilon}\left(\bar{k}_{\epsilon}, \bar{u}_{\epsilon}\right) \eta_{\epsilon}=0$ yields

$$
\dot{\mu}_{0}=\frac{4 \int_{\mathbb{R}}\left[5 \bar{u}_{\epsilon}^{2}-3\right] \bar{u}_{\epsilon} \eta_{\epsilon}^{3}}{\int_{\mathbb{R}} \eta_{\epsilon}^{2}}
$$

We were not able to find an analytical argument showing that

$$
f(\epsilon):=\int_{\mathbb{R}}\left[5 \bar{u}_{\epsilon}^{2}-3\right] \bar{u}_{\epsilon} \eta_{\epsilon}^{3}=2 \int_{0}^{\infty}\left[5 \bar{u}_{\epsilon}^{2}-3\right] \bar{u}_{\epsilon}\left|\bar{u}_{\epsilon}^{\prime}\right|^{3} \neq 0 .
$$

However, numerical computation of this integral (Fig. 1) clearly shows that it is positive for all values of $\epsilon \in(0, \sqrt{3})$,

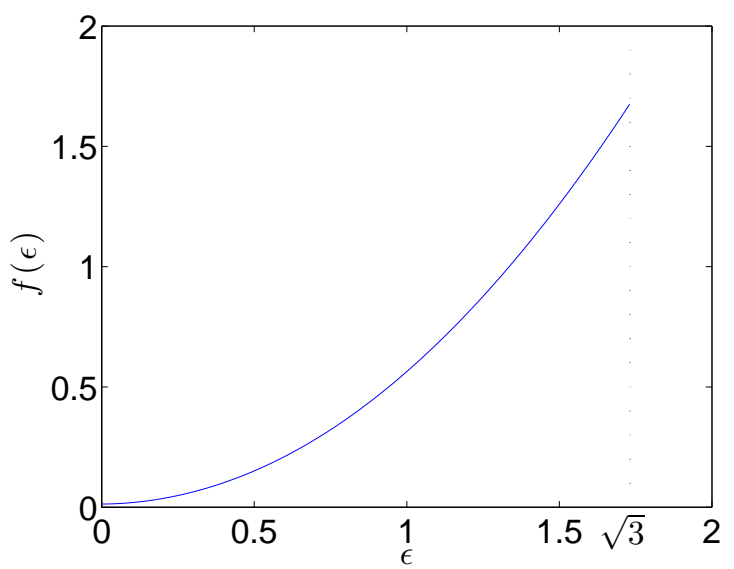

Figure 1. The graph of $f(\epsilon)$

which concludes the proof of Proposition 4

\section{Stability}

We consider the stability of the bound states

$$
\psi_{ \pm, k, \epsilon}(x, z)=\mathrm{e}^{i k z} u_{ \pm, k, \epsilon}(x)
$$

with respect to perturbations of the initial soliton profile, $u_{ \pm, k, \epsilon}$, in $H^{1}(\mathbb{R})$. Let us first remark that the Cauchy problem associated with (1.1) is globally well posed in $H^{1}(\mathbb{R})$, see $\left[7\right.$. That is, for any initial profile $\psi(\cdot, 0) \in H^{1}(\mathbb{R})$, there exists a unique continuous map $z \mapsto \psi(x, z) \in H^{1}(\mathbb{R})$, defined for all $z \in \mathbb{R}$, such that $\psi(x, z)$ satisfies (1.1).

We will now define precisely what we mean by the stability of the bound states of (1.1). It is well known that, due to the $U(1)$-invariance of (1.1), the appropriate notion of stability in this context is that of orbital stability. 
Definition 1. We say that the bound state $\psi_{k}(x, z)=\mathrm{e}^{i k z} u_{k}(x)$ is orbitally stable if

$$
\text { for all } \varepsilon>0 \text { there exists } \delta>0 \text { such that }
$$

for any solution $\varphi(x, z)$ of (1.1) with initial data $\varphi(\cdot, 0) \in H^{1}(\mathbb{R})$ there holds

$$
\left\|\varphi(\cdot, 0)-u_{k}\right\|_{H^{1}} \leqslant \delta \Longrightarrow \inf _{\theta \in \mathbb{R}}\left\|\varphi(\cdot, z)-\mathrm{e}^{i \theta} u_{k}\right\|_{H^{1}} \leqslant \varepsilon \quad \text { for all } z \geqslant 0 .
$$

Introducing the orbit $\Theta\left(u_{k}\right):=\left\{\mathrm{e}^{i \theta} u_{k}: \theta \in \mathbb{R}\right\}$ of $u_{k}$, the above statement can be rephrased as

$$
\operatorname{dist}\left(\varphi(\cdot, 0), u_{k}\right) \leqslant \delta \Longrightarrow \operatorname{dist}\left(\varphi(\cdot, z), \Theta\left(u_{k}\right)\right) \leqslant \varepsilon \text { for all } z \geqslant 0 .
$$

Here we have introduced $\operatorname{dist}(u, v):=\|u-v\|_{H^{1}}, u, v \in H^{1}(\mathbb{R})$, and the distance from a point $u \in H^{1}(\mathbb{R})$ to a set $B \subset H^{1}(\mathbb{R})$ is defined as $\operatorname{dist}(u, B):=\inf _{v \in B} \operatorname{dist}(u, v)$.

A general theory of orbital stability for Hamiltonian systems invariant under the action of a one-parameter group has been established in [17. More recently, the theory has been revisited and extended in 28] (with a special focus on nonlinear Schrödinger equations) and in [4, where it is formulated in a natural geometric framework. We shall now briefly outline how (1.1) can be interpreted as a Hamiltonian system, and state the key stability criteria we will use to prove that the whole solution curve $\mathcal{S}$ consists of orbitally stable bound states.

First, identify $H^{1}(\mathbb{R}, \mathbb{C})$ with $X:=H^{1}(\mathbb{R}, \mathbb{R}) \times H^{1}(\mathbb{R}, \mathbb{R})$ by writing $\psi=(\operatorname{Re} \psi, \operatorname{Im} \psi) \in X$ for all $\psi \in H^{1}(\mathbb{R}, \mathbb{C})$. We shall henceforth merely write $H^{1}$ for $H^{1}(\mathbb{R}, \mathbb{R})$. Identifying $L^{2}$ with its dual space, consider the variational triple $H^{1} \subset L^{2} \subset H^{-1}$, and $I: H^{1} \hookrightarrow H^{-1}$ the injection. Introducing the function $f(x, s)=\epsilon \delta(x)+2 s-s^{2}, x \in \mathbb{R}, s \geqslant 0$, (1.1) becomes

which can then be cast as

$$
i \psi_{z}=-\psi_{x x}-f\left(x,|\psi|^{2}\right) \psi
$$

$$
\frac{\mathrm{d}}{\mathrm{d} z} \psi(z)=J E^{\prime}(\psi(z))
$$

where

$$
J=\left(\begin{array}{cc}
0 & -I \\
I & 0
\end{array}\right)
$$

and the energy $E: X \rightarrow \mathbb{R}$ is given by

$$
E(\psi)=\frac{1}{2} \int_{\mathbb{R}}\left|\psi^{\prime}(x)\right|^{2} \mathrm{~d} x-\frac{1}{2} \int_{\mathbb{R}} \int_{0}^{|\psi|^{2}} f(x, s) \mathrm{d} s \mathrm{~d} x .
$$

Of course, the energy is a conserved quantity. Namely, for any solution $\psi(x, z)$ of (1.1) we have $E(\psi(\cdot, z))=E(\psi(\cdot, 0))$ for all $z \geqslant 0$. Another important conserved quantity is the power of the beam, given by

$$
Q(\psi)=\frac{1}{2} \int_{\mathbb{R}}|\psi(x)|^{2} \mathrm{~d} x
$$

For the following discussion it is important to observe that $E, Q \in C^{2}(X, \mathbb{R})$.

In this formalism, bound states take the form $\psi(x, z)=T(k z) \varphi$ for some $\varphi \in X$ and

$$
T(\theta)=\left(\begin{array}{cc}
\cos \theta & -\sin \theta \\
\sin \theta & \cos \theta
\end{array}\right), \quad \theta \in \mathbb{R} .
$$

Furthermore, the stationary equation (1.2) now reads

$$
E^{\prime}(\varphi)+k Q^{\prime}(\varphi)=0
$$

for some real $\varphi=(u, 0)$.

The Hamiltonian system (5.2) is invariant under the action of the group $\{T(\theta)\}_{\theta \in \mathbb{R}}$. This corresponds to the invariance of (1.1) with respect to multiplication by a phase factor $\mathrm{e}^{i \theta}$. It becomes apparent that the notion of orbital stability defined above is precisely stability modulo the action of this group. 
Given $k \in\left(\frac{\epsilon^{2}}{4}, \bar{k}_{\epsilon}\right]$, the orbital stability of a corresponding solution $\varphi_{k}=\left(u_{k}, 0\right)$ of (5.4) can be proved by using the Lyapunov functional $L_{k}: X \rightarrow \mathbb{R}$,

$$
L_{k}(\varphi)=E(\varphi)+k Q(\varphi) .
$$

The stability of the bound state $\varphi_{k}=\left(u_{k}, 0\right)$ then relies on a coercivity property of $L_{k}$ that can be formulated in terms of the second derivative $D^{2} L_{k}\left(\varphi_{k}\right): X \rightarrow X^{*}$, where $X^{*}=H^{-1} \times H^{-1}$. Using the stationary equation (5.4), we have

$$
D^{2} L_{k}\left(\varphi_{k}\right)=E^{\prime \prime}\left(\varphi_{k}\right)+k Q^{\prime \prime}\left(\varphi_{k}\right)=\left(\begin{array}{cc}
L^{+}\left(k, u_{k}\right) & 0 \\
0 & L^{-}\left(k, u_{k}\right)
\end{array}\right)
$$

where

$$
L^{+}\left(k, u_{k}\right)=-\frac{\mathrm{d}^{2}}{\mathrm{~d} x^{2}}+k-f\left(x, u_{k}^{2}\right)-2 \partial_{s} f\left(x, u_{k}^{2}\right) u_{k}^{2}=-\frac{\mathrm{d}^{2}}{\mathrm{~d} x^{2}}+k-\epsilon \delta(x)-6 u_{k}^{2}(x)+5 u_{k}^{4}(x)
$$

and

$$
L^{-}\left(k, u_{k}\right)=-\frac{\mathrm{d}^{2}}{\mathrm{~d} x^{2}}+k-f\left(x, u_{k}^{2}\right)=-\frac{\mathrm{d}^{2}}{\mathrm{~d} x^{2}}+k-\epsilon \delta(x)-2 u_{k}^{2}(x)+u_{k}^{4}(x) .
$$

As usual, for $v \in H^{1},-v^{\prime \prime}$ is interpreted as an element of $H^{-1}$ through $\left\langle-v^{\prime \prime}, w\right\rangle_{H^{-1} \times H^{1}}=\left\langle v^{\prime}, w^{\prime}\right\rangle_{L^{2}}$ for all $w \in H^{1}$. Various forms of the required coercivity condition are given in [28. They all express the fact that the Hessian $D^{2} L_{k}\left(\varphi_{k}\right)$ is positive definite on the codimension 2 subspace of $H^{1}$ orthogonal to the orbit $\Theta\left(u_{k}\right)$ and parallel to the tangent space to the surface $Q(u)=Q\left(u_{k}\right)$ at $u=u_{k}$; see condition (SC) in [28, p. 349] and Equ. (126) in [4. We will use here condition $\left(\mathrm{SC}^{* *}\right)$ formulated in the NLS context in [28, pp. 379-380] as follows: There exists $\delta>0$ s.t.

$$
\left\langle L^{+}\left(k, u_{k}\right) v, v\right\rangle_{H^{-1} \times H^{1}} \geqslant \delta\|v\|_{L^{2}}^{2} \text { and }\left\langle L^{-}\left(k, u_{k}\right) v, v\right\rangle_{H^{-1} \times H^{1}} \geqslant \delta\|v\|_{L^{2}}^{2} \text { for all } v \in H^{1}(\mathbb{R}, \mathbb{R}) \text { s.t. } \int_{\mathbb{R}} v u_{k} \mathrm{~d} x=0 .
$$

As earlier, we can interpret $L^{ \pm}\left(k, u_{k}\right)$ as self-adjoint operators acting in $L^{2}$. Remarking that the solution $u_{k}>0$ satisfies $L^{-}\left(k, u_{k}\right) u_{k}$, it follows by standard spectral theory [11827] that $\operatorname{ker} L^{-}\left(k, u_{k}\right)=\operatorname{span}\left\{u_{k}\right\}$, and the spectrum of $L^{-}\left(k, u_{k}\right)$ consists of the eigenvalue zero, possibly some positive eigenvalues, and the essential spectrum $[k, \infty)$. Furthermore, the spectrum of $L^{+}\left(k, u_{ \pm, k, \epsilon}\right)=T_{ \pm, k, \epsilon}$ is known from Lemma 4, and we have to distinguish three different cases:

(I) For $k \in\left(\frac{3}{4}, \bar{k}_{\epsilon}\right), L^{+}\left(k, u_{+, k, \epsilon}\right)$ has strictly positive spectrum.

(II) For $k \in\left(\frac{\epsilon^{2}}{4}, \bar{k}_{\epsilon}\right), L^{+}\left(k, u_{-, k, \epsilon}\right)$ has exactly one negative eigenvalue of multiplicity 1 , and the rest of its spectrum is strictly positive.

(III) At the fold bifurcation point, $k=\bar{k}_{\epsilon}, L^{+}\left(\bar{k}_{\epsilon}, \bar{u}_{\epsilon}\right)$ has zero as a simple eigenvalue, $\operatorname{ker} L^{+}\left(\bar{k}_{\epsilon}, \bar{u}_{\epsilon}\right)=\operatorname{span}\left\{\left|\bar{u}_{\epsilon}^{\prime}\right|\right\}$, and the rest of the spectrum is strictly positive.

The spectral scenario (II) is the most common one in the NLS literature. In this case, the coercivity condition (5.5) can be derived (see for instance [4, Proposition 9]) as a consequence of the so-called VK condition 3

$$
\frac{\mathrm{d}}{\mathrm{d} k}\left\|u_{ \pm, k, \epsilon}\right\|_{L^{2}}^{2}>0, \quad k \in\left(\frac{\epsilon^{2}}{4}, \bar{k}_{\epsilon}\right)
$$

In the physics literature, the VK condition is often regarded as a criterion for stability on its own, taking no account of the underlying spectral landscape. It may therefore seem surprising to the more physical reader that the solution curve $\mathcal{S}_{+, \epsilon}$ is indeed stable, even though it violates the VK criterion. A formal justification was nevertheless carried out by Yang in [30, where fold bifurcations for general nonlinear Schrödinger equations are studied.

We can now prove the stability theorem.

Theorem 3. Let $\epsilon \in(0, \sqrt{3})$, and $\mathcal{S}$ be defined by (3.9). Then, for all $(k, u) \in \mathcal{S}, \psi(x, z)=\mathrm{e}^{i k z} u(x)$ is an orbitally stable solution of (1.1).

\footnotetext{
${ }^{3}$ The monotonicity condition in (5.6) seems to have first been formulated by Vakhitov and Kolokolov in [29], and so is often referred to as the 'Vakhitov-Kolokolov condition' (VK condition for short).
} 
Proof. We first address cases (I) and (II), that is, the stability of the solutions belonging to the pieces of curve $\mathcal{S}_{ \pm, \epsilon}$. In case (I), the verification of (5.5) is straightforward, so $\mathcal{S}_{+, \epsilon}$ is indeed stable. For the stability of $\mathcal{S}_{-, \epsilon}$ we will prove that the function

$$
\left(\frac{\epsilon^{2}}{4}, \bar{k}_{\epsilon}\right) \ni k \mapsto\left\|u_{-, k, \epsilon}\right\|_{L^{2}}
$$

is strictly increasing. Firstly, for $k \in\left(\frac{3}{4}, \bar{k}_{\epsilon}\right)$, we find using Mathematica that

$$
\frac{\mathrm{d}}{\mathrm{d} k}\left\|u_{-, k, \epsilon}\right\|_{L^{2}}^{2}=\frac{\frac{2 \sqrt{3} \epsilon}{\sqrt{3+\epsilon^{2}-4 k}}-\frac{3}{\sqrt{k}}}{4 k-3} \text { and } \frac{\mathrm{d}}{\mathrm{d} k}\left\|u_{+, k, \epsilon}\right\|_{L^{2}}^{2}=-\frac{\frac{2 \sqrt{3} \epsilon}{\sqrt{3+\epsilon^{2}-4 k}}+\frac{3}{\sqrt{k}}}{4 k-3} .
$$

It follows that $\left\|u_{-, k, \epsilon}\right\|_{L^{2}}$ is indeed increasing, while $\left\|u_{+, k, \epsilon}\right\|_{L^{2}}$ is decreasing, for $k \in\left(\frac{3}{4}, \bar{k}_{\epsilon}\right)$. We also observe explicitly here that $\lim _{k \backslash \frac{3}{4}} \frac{\mathrm{d}}{\mathrm{d} k}\left\|u_{+, k, \epsilon}\right\|_{L^{2}}^{2}=-\infty$, which is consistent with Theorem 1 .

For $k<3 / 4$, a straightforward calculation using (2.12) shows that

$$
\left\|u_{-, k, \epsilon}\right\|_{L^{2}}^{2}=\sqrt{3} \log \varphi_{\epsilon}(k) \quad \text { where } \quad \varphi_{\epsilon}(k):=\frac{\sqrt{3} \epsilon+\sqrt{3 \epsilon^{2}+\left(4 k-\epsilon^{2}\right)(3-4 k)}+(\sqrt{3}+2 \sqrt{k})(2 \sqrt{k}-\epsilon)}{\sqrt{3} \epsilon+\sqrt{3 \epsilon^{2}+\left(4 k-\epsilon^{2}\right)(3-4 k)}+(\sqrt{3}-2 \sqrt{k})(2 \sqrt{k}-\epsilon)} .
$$

Differentiation then yields

$$
\frac{\mathrm{d}}{\mathrm{d} k} \varphi_{\epsilon}(k)=8 \sqrt{k} \frac{\sqrt{3} \sqrt{3 \epsilon^{2}+\left(4 k-\epsilon^{2}\right)(3-4 k)}+2 \sqrt{k}\left(3+\epsilon^{2}-2 \epsilon \sqrt{k}\right)}{\sqrt{3 \epsilon^{2}+\left(4 k-\epsilon^{2}\right)(3-4 k)}\left(\sqrt{3} \epsilon+\sqrt{3 \epsilon^{2}+\left(4 k-\epsilon^{2}\right)(3-4 k)}+(\sqrt{3}-2 \sqrt{k})(2 \sqrt{k}-\epsilon)\right)^{2}},
$$

where we observe that

$$
k<\frac{3}{4} \Longrightarrow 3+\epsilon^{2}-2 \epsilon \sqrt{k}>(\sqrt{3}-\epsilon)^{2}+\sqrt{3} \epsilon>0 .
$$

Therefore, $\frac{\mathrm{d}}{\mathrm{d} k} \varphi_{\epsilon}(k)>0$, so $\left\|u_{-, k, \epsilon}\right\|_{L^{2}}$ is also increasing for all $k \in\left(\frac{\epsilon^{2}}{4}, \frac{3}{4}\right)$. We have thus proved that the curves $\mathcal{S}_{ \pm, \epsilon}$ are both stable.

We finally consider case (III). To prove the stability of the solution $\left(\bar{k}_{\epsilon}, \bar{u}_{\epsilon}\right)$ we show directly that (5.5) holds. Since $\operatorname{ker} L^{-}\left(\bar{k}_{\epsilon}, \bar{u}_{\epsilon}\right)=\operatorname{span}\left\{\bar{u}_{\epsilon}\right\}$, the second condition in (5.5) is clearly satisfied. Similarly, in view of (III),

$$
\left\langle L^{+}\left(\bar{k}_{\epsilon}, \bar{u}_{\epsilon}\right) v, v\right\rangle_{H^{-1} \times H^{1}} \geqslant \delta\|v\|_{L^{2}}^{2} \text { for all } v \in H^{1}(\mathbb{R}, \mathbb{R}) \text { s.t. } \int_{\mathbb{R}} v\left|\bar{u}_{\epsilon}^{\prime}\right| \mathrm{d} x=0 .
$$

But $\int_{\mathbb{R}}\left|\bar{u}_{\epsilon}^{\prime}\right| \bar{u}_{\epsilon} \mathrm{d} x>0$, so denoting by $P$ the projection onto the orthogonal space to $\left|\bar{u}_{\epsilon}^{\prime}\right|$ in $L^{2}(\mathbb{R})$, there exists $a>0$ such that

$$
\|P v\|_{L^{2}}^{2} \geqslant a\|v\|_{L^{2}}^{2} \text { for all } v \in H^{1}(\mathbb{R}, \mathbb{R}) \text { s.t. } \int_{\mathbb{R}} v \bar{u}_{\epsilon} \mathrm{d} x=0 .
$$

Then, since $L^{+}\left(\bar{k}_{\epsilon}, \bar{u}_{\epsilon}\right): \mathcal{D}_{\epsilon} \subset L^{2}(\mathbb{R}) \rightarrow L^{2}(\mathbb{R})$ is self-adjoint with $L^{+}\left(\bar{k}_{\epsilon}, \bar{u}_{\epsilon}\right)\left|\bar{u}_{\epsilon}^{\prime}\right|=0$, it follows that

$$
\left\langle L^{+}\left(\bar{k}_{\epsilon}, \bar{u}_{\epsilon}\right) v, v\right\rangle_{H^{-1} \times H^{1}}=\left\langle L^{+}\left(\bar{k}_{\epsilon}, \bar{u}_{\epsilon}\right) P v, P v\right\rangle_{H^{-1} \times H^{1}} \geqslant \delta a\|v\|_{L^{2}}^{2} \text { for all } v \in H^{1}(\mathbb{R}, \mathbb{R}) \text { s.t. } \int_{\mathbb{R}} v \bar{u}_{\epsilon} \mathrm{d} x=0 .
$$

Hence, the second condition in (5.5) is also satisfied. The proof is complete.

Remark 3. One can deduce from (5.8) and (5.9) -(5.10) that

$$
\lim _{k \searrow 3 / 4} \frac{\mathrm{d}}{\mathrm{d} k}\left\|u_{-, k, \epsilon}\right\|_{L^{2}}^{2}=\lim _{k \succ 3 / 4} \frac{\mathrm{d}}{\mathrm{d} k}\left\|u_{-, k, \epsilon}\right\|_{L^{2}}^{2}=\sqrt{3}\left(\frac{1}{\epsilon^{2}}+\frac{1}{3}\right),
$$

showing that the slopes calculated from the solutions with $k<3 / 4$ and with $k>3 / 4$ indeed match where the two portions of $\mathcal{S}_{-, \epsilon}$ meet.

\footnotetext{
${ }^{4}$ It turns out that the expressions for $\frac{\mathrm{d}}{\mathrm{d} k}\left\|u_{ \pm, k, \epsilon}\right\|_{L^{2}}^{2}$ are much simpler than those for $\left\|u_{ \pm, k, \epsilon}\right\|_{L^{2}}^{2}$ in the regime $k \in\left(\frac{3}{4}, \bar{k}_{\epsilon}\right)$.
} 


\section{NuMERICS}

Hereafter we present a numerical method, which we used for computing solutions of Eq. (1.2). This was helpful to understand the behavior of solutions before we had found their explicit representations. The method is based on the continuous normalized gradient flow, which was studied and implemented in [2] in the context of the NLS equation with a cubic nonlinearity.

6.1. The numerical scheme. We look for a minimizer of the energy

$$
E(u)=\frac{1}{2}\left\{\left\|u_{x}\right\|_{L^{2}}^{2}-\epsilon|u(0)|^{2}-\|u\|_{L^{4}}^{4}+\frac{1}{3}\|u\|_{L^{6}}^{6}\right\},
$$

with a given power constraint

$$
\|u\|_{L^{2}}=a>0
$$

The minimizer is then a solution of (1.2) which can be interpreted as a (nonlinear) eigenfunction with eigenvalue

$$
k=\frac{-\left\|u_{x}\right\|_{L^{2}}^{2}+\epsilon|u(0)|^{2}+2\|u\|_{L^{4}}^{4}-\|u\|_{L^{6}}^{6}}{\|u\|_{L^{2}}^{2}} .
$$

In the physics literature, this method is known as imaginary time propagation $(z \rightarrow-i t)[8$

$$
u_{t}=-\frac{\delta E(u)}{\delta u}=u_{x x}+\epsilon \delta(x) u+2|u|^{2} u-|u|^{4} u .
$$

Thus, in order to solve (1.2), we introduce the imaginary time and iterate in this time. After each time step, we renormalize the solution so as to maintain the constraint (6.2). The discretization of (6.1) is done by means of semi-implicit backward Euler central differences.

Let us consider the time sequence $t_{0}<t_{1}<t_{2}<\cdots<t_{n}$, with time step $\mathrm{d} t=t_{n}-t_{n-1}$, and space grid $x_{j}=x_{0}+j h_{x}$ with $j=0,1,2, \ldots, J$, where we solve the equation on $\left[x_{0}, x_{J}\right]$ with $J$ grid points and the mesh size $h_{x}=\left(x_{J}-x_{0}\right) / J$. The discrete solution is denoted by $u_{j}^{n}=u\left(t^{n}, x_{j}\right)$ and $j_{0}$ is the index for which $x_{j_{0}}=0$. At $x_{j_{0}}$ we use the properties of $u$ in Proposition 1(v), in the discrete form:

$$
u_{j_{0}+1}^{n}=\left(1-\frac{h_{x} \cdot \epsilon}{2}\right) u_{j_{0}}^{n}, \quad u_{j_{0}-1}^{n}=\left(1-\frac{h_{x} \cdot \epsilon}{2}\right) u_{j_{0}}^{n}
$$

On $\left[t_{n}, t_{n+1}\right]$ we solve:

$$
\begin{array}{rlrl}
\frac{u_{j}^{*}-u_{j}^{n}}{\mathrm{~d} t} & =\frac{u_{j+1}^{*}-2 u_{j}^{*}+u_{j-1}^{*}}{h_{x}^{2}}+2\left(u_{j}^{n}\right)^{2} u_{j}^{*}-\left(u_{j}^{n}\right)^{4} u_{j}^{*} & \text { for } 0 \leqslant j<j_{0}-1 \text { and } j_{0}+1<j \leqslant J ; \\
\frac{u_{j}^{*}-u_{j}^{n}}{\mathrm{~d} t}=\frac{u_{j}^{*}\left(\frac{2}{2-h x \cdot \epsilon}-2\right)+u_{j}^{*}}{h_{x}^{2}}+2\left(u_{j}^{n}\right)^{2} u_{j}^{*}-\left(u_{j}^{n}\right)^{4} u_{j}^{*} & \text { for } j=j_{0}-1 ; \\
u_{j_{0}}^{*}=\frac{u_{j_{0}-1}^{*}}{\left(1-\frac{h_{x} \cdot \epsilon}{2}\right)} & \text { for } j=j_{0} ; \\
\frac{u_{j}^{*}-u_{j}^{n}}{\mathrm{~d} t}=\frac{u_{j+1}^{*}+u_{j}^{*}\left(\frac{2}{2-h_{x} \cdot \epsilon}-2\right)}{h_{x}^{2}}+2\left(u_{j}^{n}\right)^{2} u_{j}^{*}-\left(u_{j}^{n}\right)^{4} u_{j}^{*} & \text { for } j=j_{0}+1 ; \\
u_{j}^{n+1} & =\frac{a \cdot u_{j}^{*}}{\left\|u^{*}\right\|_{2}} & \text { for all } j .
\end{array}
$$

6.2. Numerical simulations. In this section we compare the discretized solution $u_{j}^{n}$ with the exact one for different values of the parameters. We solve the equation on [-40,40], with $J=3200$ grid points and time step $\mathrm{d} t=10^{-4}$ (thus $x_{0}=-40, x_{J}=40, j_{0}=1600$ and $h_{x}=1 / 40$ ). For fixed $\epsilon$ we draw the bifurcation diagram for the power of the exact solution (2.12)-(2.14), i.e., its norm $\|u\|_{L^{2}}$, and pick up values $a_{1}, a_{2}, \ldots, a_{6}$ of the power, see Fig. 3 . Then we calculate the discretized solution $u_{j}^{n}$ with fixed power $a_{l}(l=1,2, \ldots, 6)$, and compare it to the exact solution with the corresponding $k$. 


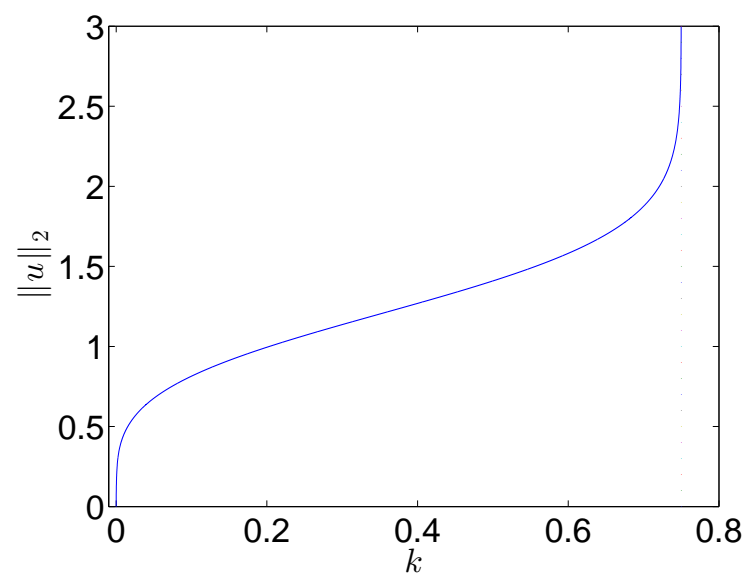

FIgURE 2. For $\epsilon=0$, we plot $\|u\|_{L^{2}}$ against $k$, using the explicit solutions $u_{-, k, 0}$ obtained in Section 2
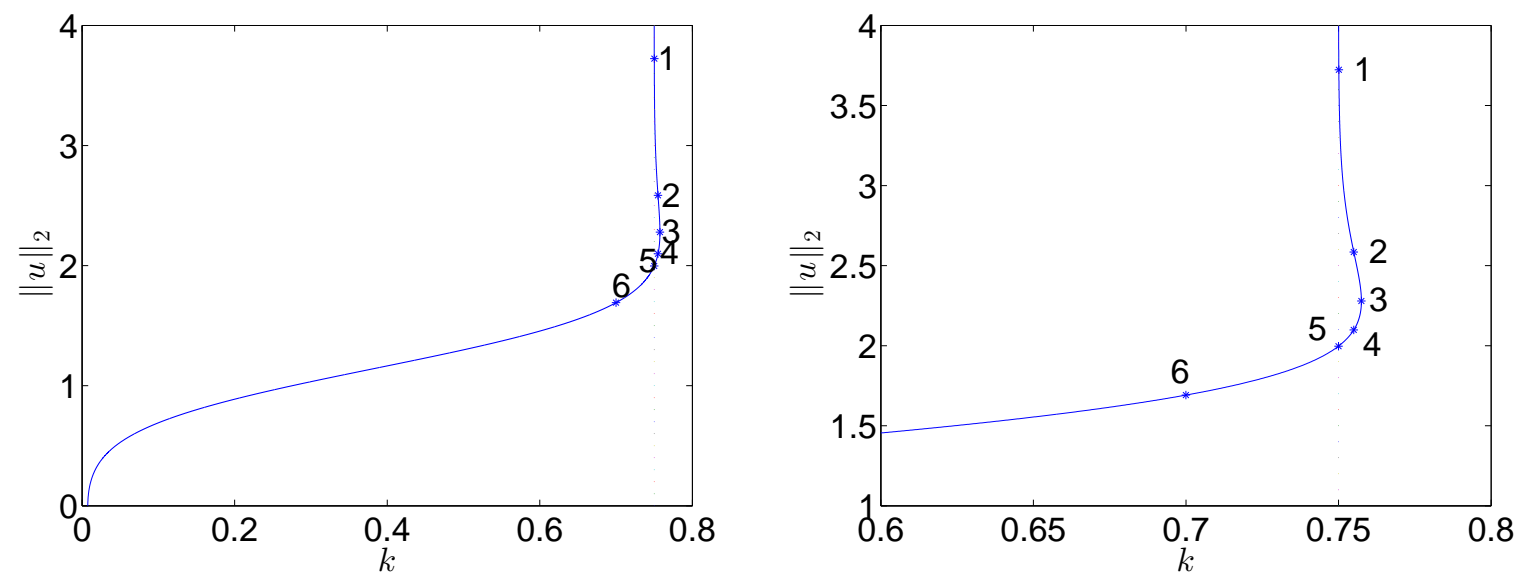

Figure 3. For $\epsilon=0.1 \sqrt{3}$, we plot $\|u\|_{L^{2}}$ against $k$, using the explicit solutions $u_{ \pm, k, \epsilon}$ obtained in Section 2, In the second plot we zoomed in, to have a closer view of the fold bifurcation.

In Fig. 2 the bifurcation diagram for the $L^{2}$ norm of $u_{-, k, 0}$ is displayed for $\epsilon=0$ and $k \in\left(0, \frac{3}{4}\right)$. Fig. (3) 5 illustrate the bifurcation diagrams for different values of $\epsilon>0$, namely $0.1 \sqrt{3}, 0.5 \sqrt{3}$ and $0.9 \sqrt{3}$. We plot the $L^{2}$ norm of the solutions against $k$. The lower branches are obtained from $u_{-, k, \epsilon}$ with $k \in\left(\frac{\epsilon^{2}}{4}, \bar{k}_{\epsilon}\right)$, while the upper branches display the $L^{2}$ norm of $u_{+, k, \epsilon}$ with $k \in\left(\frac{3}{4}, \bar{k}_{\epsilon}\right)$. In each diagram we observe the behavior predicted by the exact analysis of the previous sections: the $L^{2}$ norm bifurcates from zero at $k=\frac{\epsilon^{2}}{4}$ and diverges along the upper branch as $k \searrow \frac{3}{4}$, after 'turning backwards' at $k=\bar{k}_{\epsilon}$.

In Fig. 3. for $\epsilon=0.1 \sqrt{3}$, we pick up six different values $a_{1}, \ldots, a_{6}$ of the $L^{2}$ norm for which we then compare, in Fig. 6, the exact solutions $u_{ \pm, k, \epsilon}$ to the numerical solutions $u^{n}\left(x_{j}\right)$ obtained by minimization under the constraints $a_{1}$ to $a_{6}$; see plots 1) -6) in Fig. 6. In contrast to the analytical computation of the solution, where we had to distinguish the two branches $u_{ \pm, k, \epsilon}$ in the range $k \in\left(\frac{3}{4}, \bar{k}_{\epsilon}\right)$, the numerical computation is performed at fixed $L^{2}$ norm, and provides the only solution corresponding to each given value $a=\|u\|_{L^{2}}$. 


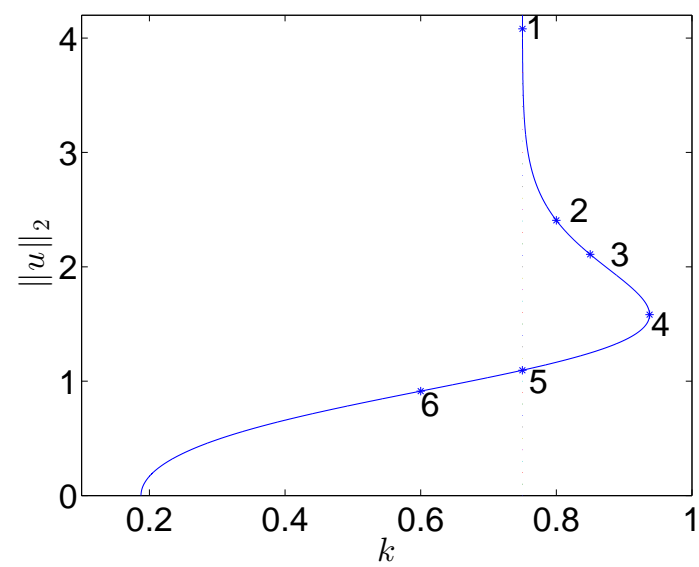

Figure 4. For $\epsilon=0.5 \sqrt{3}$, we plot $\|u\|_{L^{2}}$ against $k$, using the explicit solutions $u_{ \pm, k, \epsilon}$ obtained in Section 2 ,

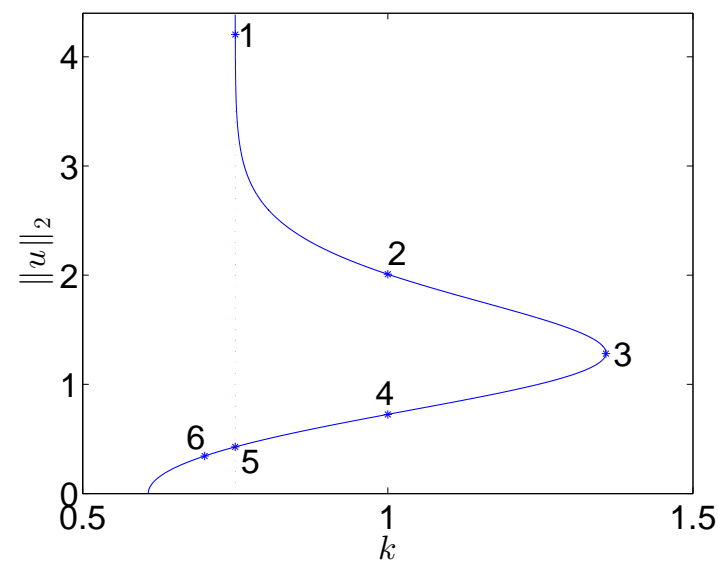

Figure 5. For $\epsilon=0.9 \sqrt{3}$, we plot $\|u\|_{L^{2}}$ against $k$, using the explicit solutions $u_{ \pm, k, \epsilon}$ obtained in Section 2 .

Similarly, in Fig. 7 and Fig. 8 we compare the exact solutions with the numerical ones, for $\epsilon=0.5 \sqrt{3}$ and $\epsilon=0.9 \sqrt{3}$, respectively. We again use six values of the $L^{2}$ norm taken from the corresponding bifurcation diagrams in Fig. 4 and Fig. 5. In all three cases we notice an excellent agreement between the exact solutions and the numerical ones. So far, the continuous normalized gradient flow (CNGF) has been mostly used for the NLS with a cubic nonlinearity. Our results demonstrate its effectiveness in the case of a cubic-quintic nonlinearity. Furthermore, the CNGF being variational in nature, this suggests that the positive solutions of (1.2) should admit a variational characterization as obtained for instance in [14] in the case of a single power nonlinearity. This will be discussed further elsewhere. 
1)
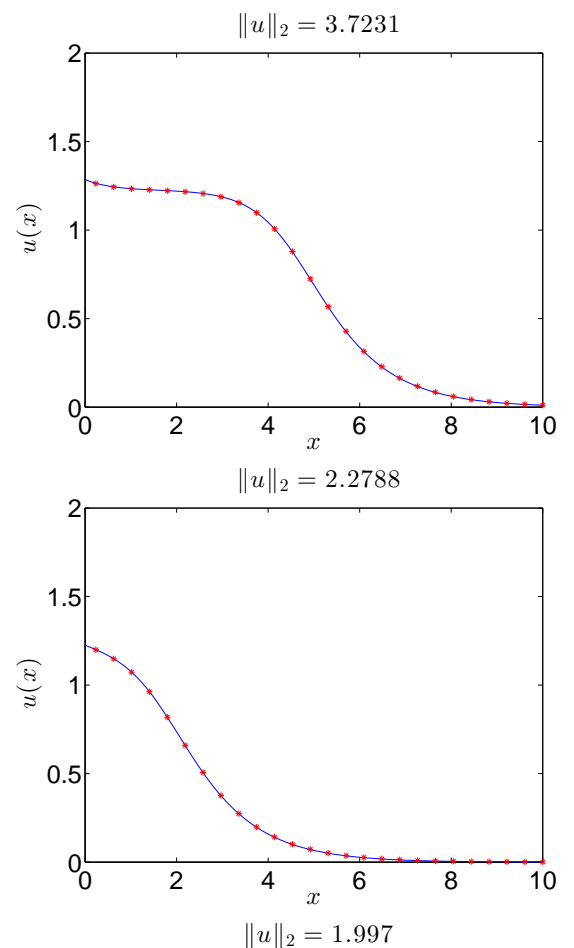

3)

4)

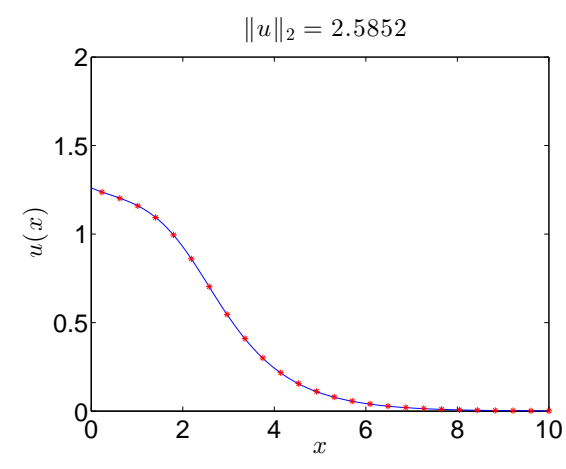

2)

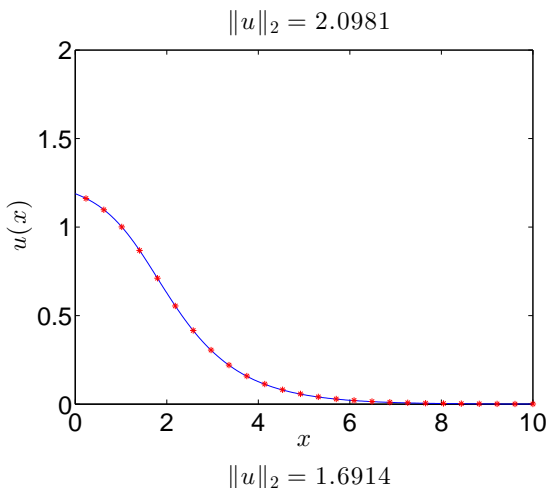

$6)$

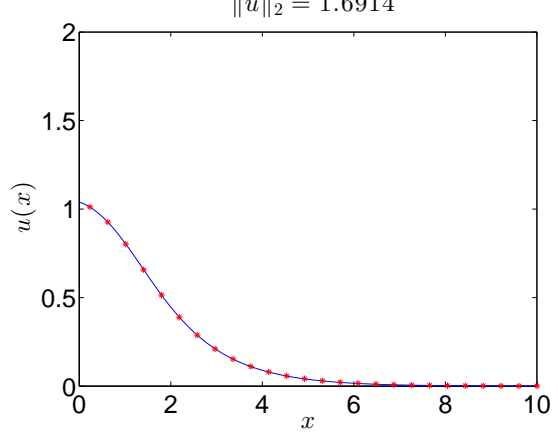

Figure 6 . For $\epsilon=0.1 \sqrt{3}$, in each of the plots 1)-6) we compare the discrete solution $u^{n}\left(x_{j}\right)(*)$ to the exact solution $u_{ \pm, k, \epsilon}(x)$ (solid line), corresponding to the points on the bifurcation curve in Fig. 3 . 
1)

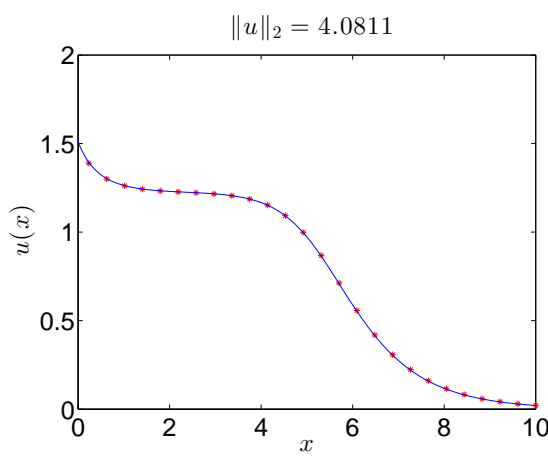

3)
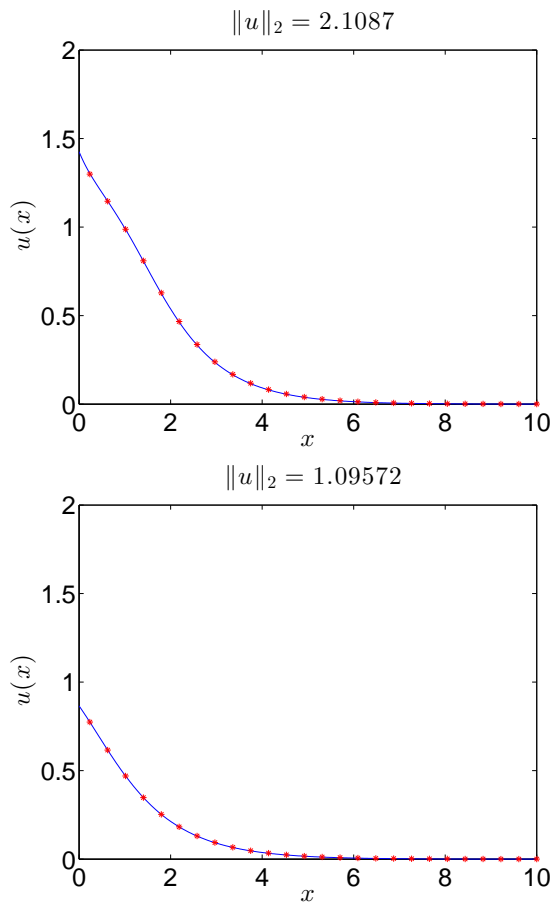

2)

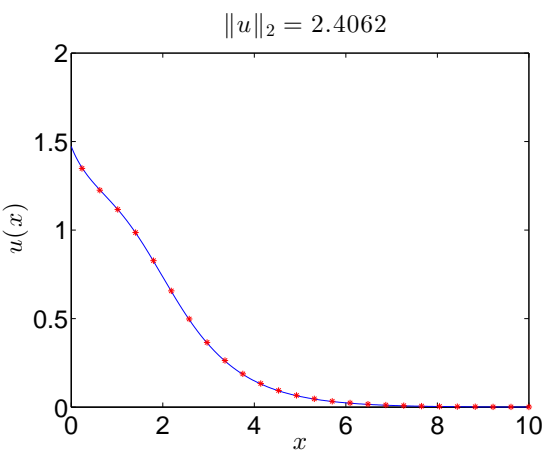

4)
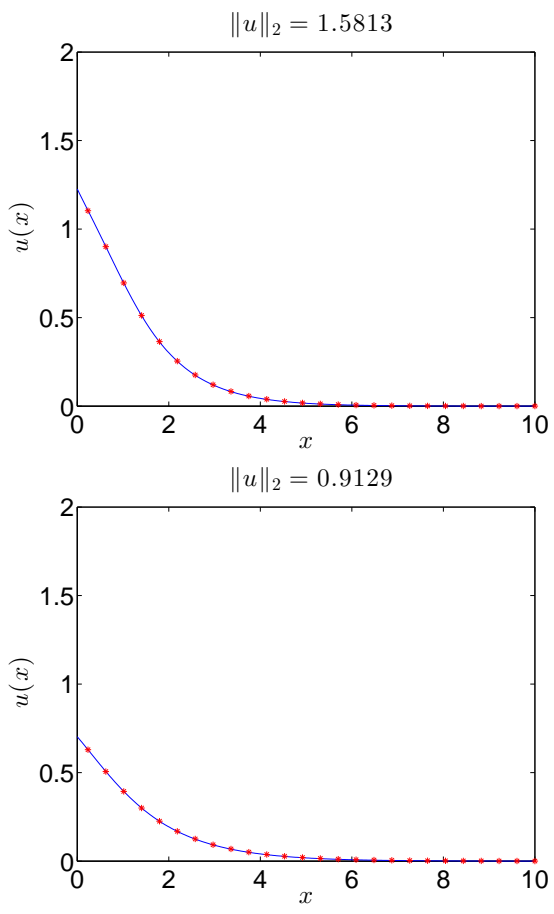

Figure 7 . For $\epsilon=0.5 \sqrt{3}$, in each of the plots 1)-6) we compare the discrete solution $u^{n}\left(x_{j}\right)(*)$ to the exact solution $u_{ \pm, k, \epsilon}(x)$ (solid line), corresponding to the points on the bifurcation curve in Fig. 固 
1)

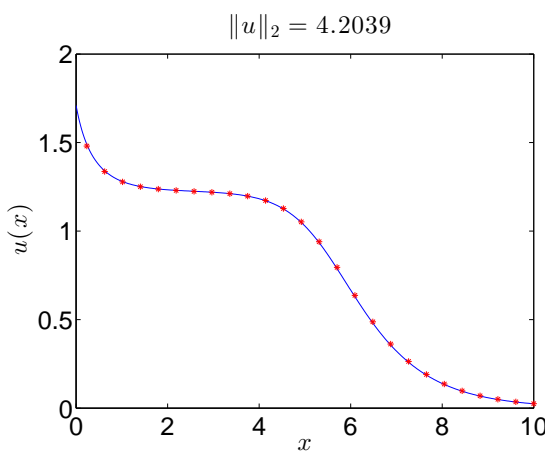

$\|u\|_{2}=1.282$

$3)$

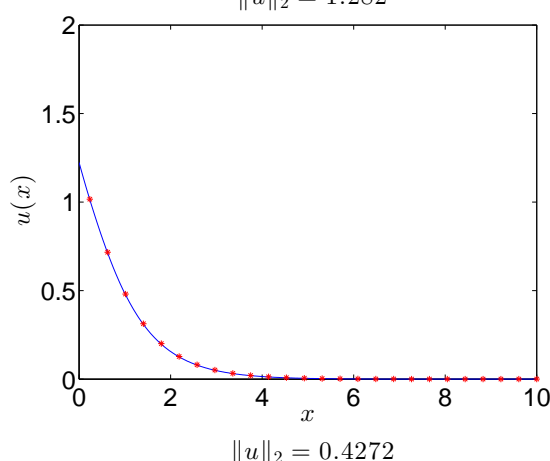

$5)$

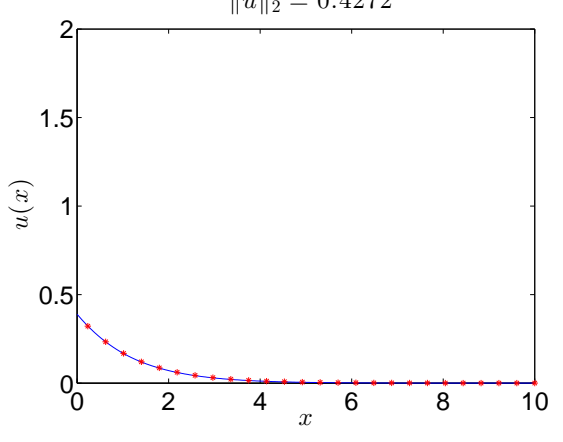

2)

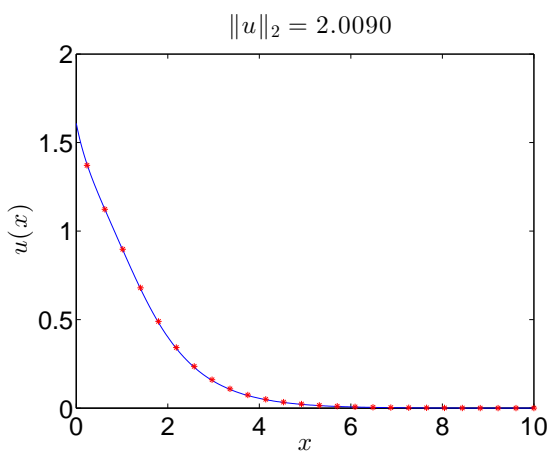

4)

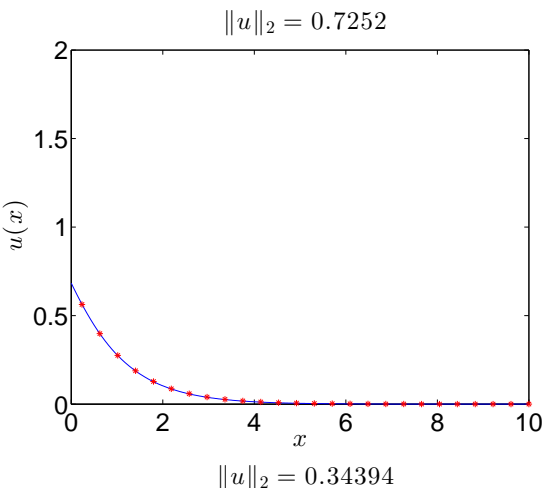

6)

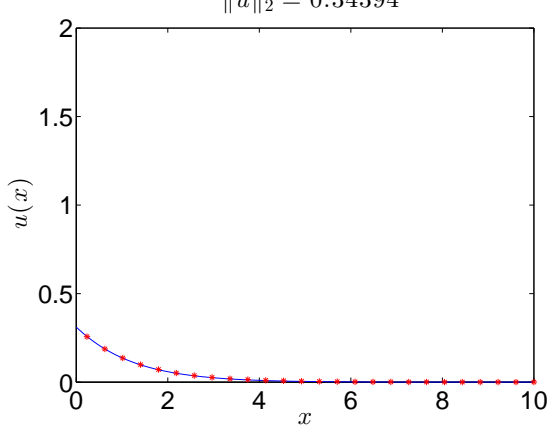

Figure 8 . For $\epsilon=0.9 \sqrt{3}$, in each of the plots 1)-6) we compare the discrete solution $u^{n}\left(x_{j}\right)(*)$ to the exact solution $u_{ \pm, k, \epsilon}(x)$ (solid line), corresponding to the points on the bifurcation curve in Fig. 5 . 


\section{REFERENCES}

[1] S. Albeverio, F. Gesztesy, R. Høegh-Krohn, H. Holden, Solvable Models in Quantum Mechanics, Second edition, AMS Chelsea Publishing, Providence, RI, 2005.

[2] W. Bao, Q. Du, Computing the ground state solution of Bose-Einstein condensates by a normalized gradient flow, SIAM J. Sci. Comput., 25 (2004), 1674-1697.

[3] F. A. Berezin, M. A. Shubin, The Schrödinger Equation, Kluwer Academic Publishers, 1991.

[4] S. de Bièvre, F. Genoud, S. Rota-Nodari, Orbital stability: analysis meets geometry, in: C. Besse, J. C. Garreau (eds.), Nonlinear Optical and Atomic Systems, Lecture Notes in Mathematics 2146, Springer, 2015, pp. 147-273.

[5] Z. Birnbaum, B. A. Malomed, Families of spatial solitons in a two-channel waveguide with the cubic-quintic nonlinearity, Phys. D 237 (2008), 3252-3262.

[6] G. Boudebs, S. Cherukulappurath, H. Leblond, J. Troles, F. Smektala, F. Sanchez, Experimental and theoretical study of higher-order nonlinearities in chalcogenide glasses, Opt. Commun. 219 (2003), 427-433.

[7] T. Cazenave, Semilinear Schrödinger Equations, Courant Lecture Notes in Mathematics, American Mathematical Society, Providence, RI, 2003.

[8] M. L. Chiofalo, S. Succi, M. P. Tosi, Ground state of trapped interacting Bose-Einstein condensates by an explicit imaginary-time algorithm, Phys. Rev. E 62 (2000), 7438-7444.

[9] S. Cowan, R. H. Enns, S. S. Rangnekar, S. S. Sanghera, Quasi-soliton and other behavior of the nonlinear cubic-quintic Schrödinger equation, Can. J. Phys. 64 (1986), 311-315.

[10] M. G. Crandall, P. H. Rabinowitz, Bifurcation from simple eigenvalues, J. Functional Analysis 8 (1971), 321-340.

[11] M. G. Crandall, P. H. Rabinowitz, Bifurcation, perturbation of simple eigenvalues and linearized stability, Arch. Rational Mech. Anal. 52 (1973), 161-180.

[12] E. L. Falcão-Filho, C. B. de Araújo, J. J. Rodrigues Jr., High-order nonlinearities of aqueous colloids containing silver nanoparticles, J. Opt. Soc. Am. B 24 (2007), 2948-2956.

[13] E. L. Falcão-Filho, C. B. de Araújo, G. Boudebs, H. Leblond, V. Skarka, Robust two-dimensional spatial solitons in liquid carbon disulfide, Phys. Rev. Lett. 110 (2013), 013901.

[14] R. Fukuizumi, L. Jeanjean, Stability of standing waves for a nonlinear Schrödinger equation with a repulsive Dirac delta potential, Discrete Contin. Dyn. Syst. 21 (2008), 121-136.

[15] R. Fukuizumi, M. Ohta, T. Ozawa, Nonlinear Schrödinger equation with a point defect, Ann. Inst. H. Poincaré Anal. Non Linéaire 25 (2008), 837-845.

[16] B. V. Gisin, R. Driben, B. A. Malomed, Bistable guided solitons in the cubic-quintic medium, J. Optics B: Quantum and Semiclassical Optics 6 (2004), S259-S264.

[17] M. Grillakis, J. Shatah, W. Strauss, Stability theory of solitary waves in the presence of symmetry I, J. Funct. Anal., 74 (1987), 160-197.

[18] T. Kato, Perturbation Theory for Linear Operators, Reprint of the 1980 edition, Classics in Mathematics, Springer-Verlag, Berlin, 1995.

[19] S. Le Coz, R. Fukuizumi, G. Fibich, B. Ksherim, Y. Sivan, Instability of bound states of a nonlinear Schrödinger equation with a Dirac potential, Phys. D $\mathbf{2 3 7}$ (2008), 1103-1128.

[20] M. Maeda, Stability and instability of standing waves for 1-dimensional nonlinear Schrödinger equation with multiple-power nonlinearity, Kodai Math. J. 31 (2008), 263-271.

[21] B. A. Malomed, M. Ya. Azbel, Modulational instability of a wave scattered by a nonlinear center, Phys. Rev. B 47 (1993), $10402-10406$.

[22] M. Ohta, Stability and instability of standing waves for one-dimensional nonlinear Schrödinger equations with double power nonlinearity, Kodai Math. J. 18 (1995), 68-74.

[23] P. Papagiannis, Y. Kominis, K. Hizanidis, Power- and momentum-dependent soliton dynamics in lattices with longitudinal modulation, Phys. Rev. A 84 (2011), 013820.

[24] D. E. Pelinovsky, Y. S. Kivshar, V. V. Afanasjev, Internal modes of envelope solitons, Physica D 116 (1998), $121-142$.

[25] K. I. Pushkarov, D. I. Pushkarov, I. V. Tomov, Self-action of light beams in nonlinear media: soliton solutions, Opt. Quantum Electron. 11 (1979) 471-478.

[26] P. H. Rabinowitz, Some global results for nonlinear eigenvalue problems, J. Functional Analysis 7 (1971), $487-513$.

[27] C. A. Stuart, An introduction to elliptic equations on $R^{N}$, in: Nonlinear Functional Analysis and Applications to Differential Equations, Trieste, 1997, World Sci. Publ., River Edge, NJ, 1998, pp. 237-285.

[28] C. A. Stuart, Lectures on the orbital stability of standing waves and application to the nonlinear Schrödinger equation, Milan J. Math. 76 (2008), 329-399.

[29] N. G. Vakhitov, A. A. Kolokolov, Stationary solutions of the wave equation in a medium with nonlinearity saturation, Radiophys. Quantum Electron., 16 (1973), 783-789.

[30] J. Yang, Conditions and stability analysis for saddle-node bifurcations of solitary waves in generalized nonlinear Schrödinger equations, in: B. A. Malomed (ed.), Spontaneous Symmetry Breaking, Self-Trapping, and Josephson Oscillations, Progress Optical Sci., Photonics 1 (2013), pp. 639-655. 
[31] J. Yang, Stability switching at transcritical bifurcations of solitary waves in generalized nonlinear Schrödinger equations, Phys. Lett. A 377 (2013), 866-870.

[32] K. B. Zegadlo, T. Wasak, B. A. Malomed, M. A. Karpierz, M. Trippenbach, Stabilization of solitons under competing nonlinearities by external potentials, Chaos 24 (2014), 043136.

Faculty of Mathematics, University of Vienna, Oskar-Morgenstern-Platz 1, 1090 Vienna, Austria

Current address: Delft Institute of Applied Mathematics, Delft University of Technology, Mekelweg 4, 2628 CD Delft, The Netherlands

E-mail address: S.F.Genoud@tudelft.nl

Department of Physical Electronics, School of Electrical Engineering, Faculty of Engineering, Tel Aviv University, Tel Aviv 69978, IsRael

E-mail address: malomed@post.tau.ac.il

Faculty of Mathematics, University of Vienna, Oskar-Morgenstern-Platz 1, 1090 Vienna, Austria

E-mail address: rada.weishaeupl@univie.ac.at 